\title{
Article \\ IL-25 Induced ROS-Mediated M2 Macrophage Polarization via AMPK-Associated Mitophagy
}

\author{
Mei-Lan Tsai ${ }^{1,2}$, Yi-Giien Tsai ${ }^{3,4,5}$, Yu-Chih Lin ${ }^{6,7} \mathbb{1}$, Ya-Ling Hsu ${ }^{1,8}$, Yi-Ting Chen ${ }^{2}$, Ming-Kai Tsai ${ }^{9}$, \\ Wei-Ting Liao ${ }^{10,11, *,+}(\mathbb{D}$, Yi-Ching Lin $10,12,13,14, *,+(\mathbb{D}$ and Chih-Hsing Hung $1,2,15,16,17, *,+(\mathbb{D}$
}

1 Graduate Institute of Medicine, College of Medicine, Kaohsiung Medical University, Kaohsiung 807, Taiwan; snoopy905@gmail.com (M.-L.T.); yainghsu@kmu.edu.tw (Y.-L.H.)

2 Department of Pediatrics, Faculty of Pediatrics, College of Medicine, Kaohsiung Medical University, Kaohsiung 807, Taiwan; pinkkitty1121@gmail.com

3 Department of Pediatrics, Changhua Christian Children Hospital, Changhua 500, Taiwan; 107239@cch.org.tw

4 School of Medicine, Kaohsiung Medical University, Kaohsiung 807, Taiwan

5 School of Medicine, Chung Shan Medical University, Taichung 402, Taiwan

6 Department of Medical Humanities and Education, School of Medicine, Kaohsiung Medical University, Kaohsiung 807, Taiwan; springfred@gmail.com

7 Department of Internal Medicine, Division of Allergology, Immunology and Rheumatology, Kaohsiung Medical University, Kaohsiung 807, Taiwan

8 Drug Development and Value Creation Research Center, Kaohsiung Medical University, Kaohsiung 807, Taiwan

9 Department of Internal Medicine, Division of Nephrology, Kaohsiung Armed Forces General Hospital, Kaohsiung 802, Taiwan; tmk802@gmail.com

10 Department of Medical Research, Kaohsiung Medical University Hospital, Kaohsiung Medical University, Kaohsiung 807, Taiwan

11 Department of Biotechnology, College of Life Science, Kaohsiung Medical University, Kaohsiung 807, Taiwan

12 Department of Laboratory Medicine, Kaohsiung Medical University Hospital, Kaohsiung Medical University, Kaohsiung 807, Taiwan

check for
updates

Citation: Tsai, M.-L.; Tsai, Y.-G. Lin, Y.-C.; Hsu, Y.-L.; Chen, Y.-T.; Tsai, M.-K.; Liao, W.-T.; Lin, Y.-C.; Hung, C.-H. IL-25 Induced ROS-Mediated M2 Macrophage

Polarization via AMPK-Associated Mitophagy. Int. J. Mol. Sci. 2022, 23, 3 . https://doi.org/10.3390/ijms23010003

Academic Editor: Narasimham L. Parinandi

Received: 14 October 2021 Accepted: 18 December 2021 Published: 21 December 2021

Publisher's Note: MDPI stays neutral with regard to jurisdictional claims in published maps and institutional affiliations.

Copyright: (c) 2021 by the authors. Licensee MDPI, Basel, Switzerland This article is an open access article distributed under the terms and conditions of the Creative Commons Attribution (CC BY) license (https:// creativecommons.org/licenses/by/ $4.0 /)$.
13 Doctoral Degree Program of Toxicology, College of Pharmacy, Kaohsiung Medical University, Kaohsiung 807, Taiwan

14 Department of Laboratory Medicine, School of Medicine, College of Medicine, Kaohsiung Medical University, Kaohsiung 807, Taiwan

15 Department of Pediatrics, Kaohsiung Medical University Hospital, Kaohsiung Medical University, Kaohsiung 807, Taiwan

16 Research Center for Environmental Medicine, Kaohsiung Medical University, Kaohsiung 807, Taiwan

17 Department of Pediatrics, Kaohsiung Municipal Siaogang Hospital, Kaohsiung 812, Taiwan

* Correspondence: wtliao@kmu.edu.tw (W.-T.L.); winterjeanne@gmail.com (Y.-C.L.); pedhung@gmail.com (C.-H.H.); Tel.: +886-7-311-5140 (C.-H.H.); Fax: +886-7-321-3931 (C.-H.H.)

+ The authors Wei-Ting Liao, Yi-Ching Lin, and Chih-Hsing Hung contributed equally to this work.

\begin{abstract}
Interleukin (IL)-25 is a cytokine released by airway epithelial cells responding to pathogens. Excessive production of reactive oxygen species (ROS) leads to airway inflammation and remodeling in asthma. Mitochondria are the major source of ROS. After stress, defective mitochondria often undergo selective degradation, known as mitophagy. In this study, we examined the effects of IL-25 on ROS production and mitophagy and investigated the underlying mechanisms. The human monocyte cell line was pretreated with IL-25 at different time points. ROS production was measured by flow cytometry. The involvement of mitochondrial activity in the effects of IL-25 on ROS production and subsequent mitophagy was evaluated by enzyme-linked immunosorbent assay, Western blotting, and confocal microscopy. IL-25 stimulation alone induced ROS production and was suppressed by N-acetylcysteine, vitamin C, antimycin A, and MitoTEMPO. The activity of mitochondrial complex I and complex II/III and the levels of p-AMPK and the mitophagy-related proteins were increased by IL-25 stimulation. The CCL-22 secretion was increased by IL-25 stimulation and suppressed by mitophagy inhibitor treatment and PINK1 knockdown. The Th2-like cytokine IL-25 can induce ROS production, increase mitochondrial respiratory chain complex activity, subsequently activate AMPK, and induce mitophagy to stimulate M2 macrophage polarization in monocytes.
\end{abstract}


Keywords: IL-25; reactive oxygen species; AMPK; mitophagy; M2 macrophage polarization

\section{Introduction}

Interleukin (IL)-25 is an epithelial-derived cytokine that links innate and adaptive immunity by enhancing Th2 responses [1,2]. IL-25 is produced by immune cells and epithelial cells [3]. It regulates the internal safety of adaptive immune responses, leading to the initiation of allergic diseases and playing a role in stimulating of pulmonary mucosal cells and fibroblasts [1,4]. IL-25 production in humans and mice or injection of IL-25 into animals has been shown to result in the secretion of high levels of Th2 cytokines, such as IL-4, IL-5, and IL-13 [5]. Pilot studies have shown that IL-25 mRNA is highly expressed in Th2 cells. Furthermore, in a murine pulmonary fibrosis model, collagen deposition in the lungs of challenged mice was driven by IL-25-induced type 2 innate lymphoid cells (ILC2s)-released IL-13 [6]. Therefore, IL-25 and ILC2s could be therapeutic targets of human fibrotic diseases.

The physiological damage caused by oxidative stress resulting from reactive oxygen species (ROS) has been demonstrated to induce airway hyperresponsiveness (AHR) by smooth muscle contraction and enhance mucus secretion and epithelial shedding [7]. Increased generation of ROS has been demonstrated in patients with asthma. Overexpression of ROS leads to oxidative stress, contributing to the emergence and persistence of pulmonary fibrosis induced by TGF- $\beta$. ROS are considered to be vital mediators of pulmonary vascular cell proliferation and enhance VEGF expression, which increases the thickness of pulmonary arterial walls and promotes vascular remodeling [8].

Mitochondria-specific autophagy is called mitophagy and occurs in defective mitochondria after damage or stress. The dysregulation of mitophagy plays an important role in the development of chronic diseases, including neurodegenerative diseases, metabolic diseases, and heart failure [9]. PTEN-induced putative kinase 1 (PINK1) is a serine/threonine kinase that contains a mitochondrial targeting sequence, and Parkin is required for mitophagy induction. The pathways of mitophagy initiation overlap considerably with those required for general autophagy [10] and have been shown to contribute to pulmonary fibrosis [11]. Thus, ROS production in structural lung cells, such as epithelial cells, is also likely to play an important role [12]. IL-25 is a Th2-like cytokine, and several studies have suggested its relationship with asthma [13]. However, the detailed mechanisms underlying the effect of IL-25 on ROS production remain unclear. In the present study, we investigated the effects of IL-25 on ROS production and the possible underlying mechanisms in human monocytes.

\section{Results}

The Th2-like cytokine IL-25 induced intracellular ROS production in THP-1 cells and THP-1 derived macrophages.

IL-25 is defined as an upstream epithelial cytokine driving the production of potent Th2 cytokines, such as IL-5 and IL-13, which have been proven to be involved in severe, difficultto-treat eosinophilic asthma. Therefore, we first examined the effect of another Th2-like cytokine, IL-25, on ROS production in THP-1 cells and THP-1 derived macrophages. IL-25 (2-40 ng/mL) induced ROS production in THP-1 cells and THP-1 derived macrophages at the $0.5 \mathrm{~h}$ (Figure 1A) and $2 \mathrm{~h}$ (Figure 1B) time points. We selected $2 \mathrm{~h}$ as a detection time point to investigate the antioxidative effect on IL-25-induced ROS production. The IL-25induced ROS production in THP-1 cells and THP-1 derived macrophages were significantly inhibited by NAC, vitamin C (Figure 1C). Moreover, we further used mitochondrial complex III inhibitor antimycin A and mitochondrial-targeting antioxidant MitoTEMPO to examine the mitochondria-specific antioxidative effect. The IL-25-induced ROS production in THP-1 cells and THP-1 derived macrophages were also significantly suppressed by antimycin A (Figure 1D) and MitoTEMPO (Figure 1E). 
(A)
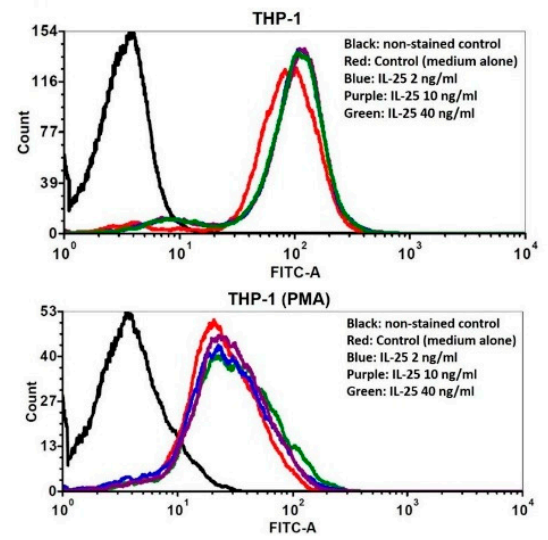

$0.5 \mathrm{~h}$

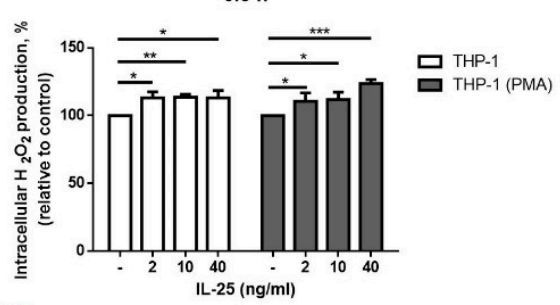

(D)
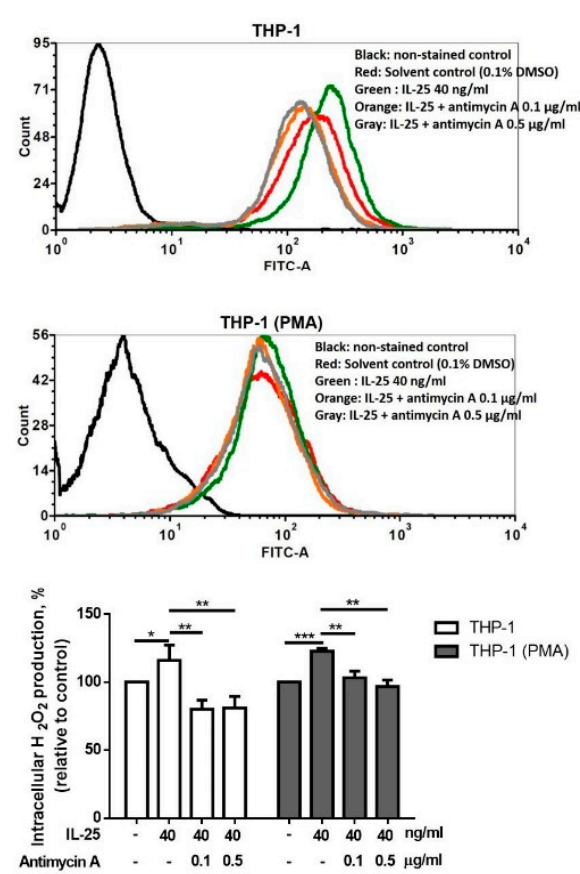

(B)
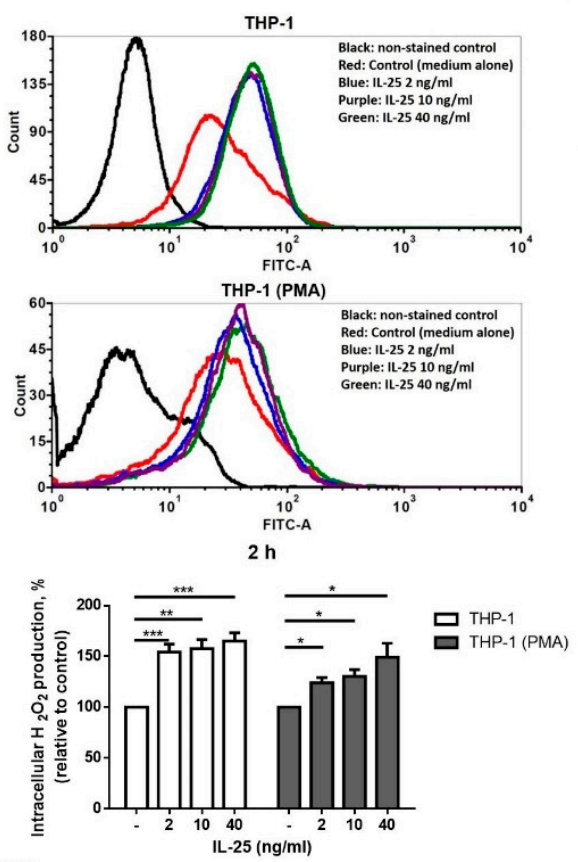

(E)
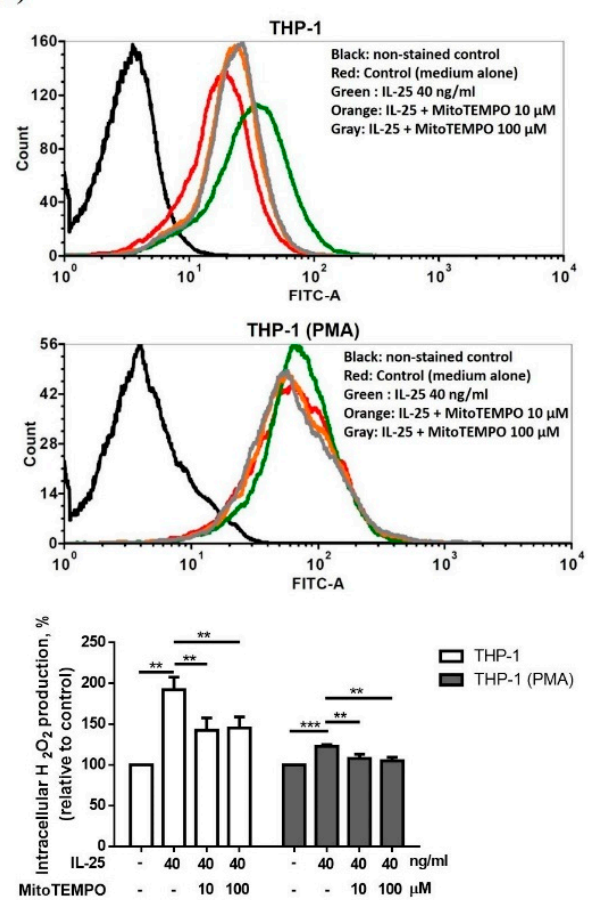

(C)
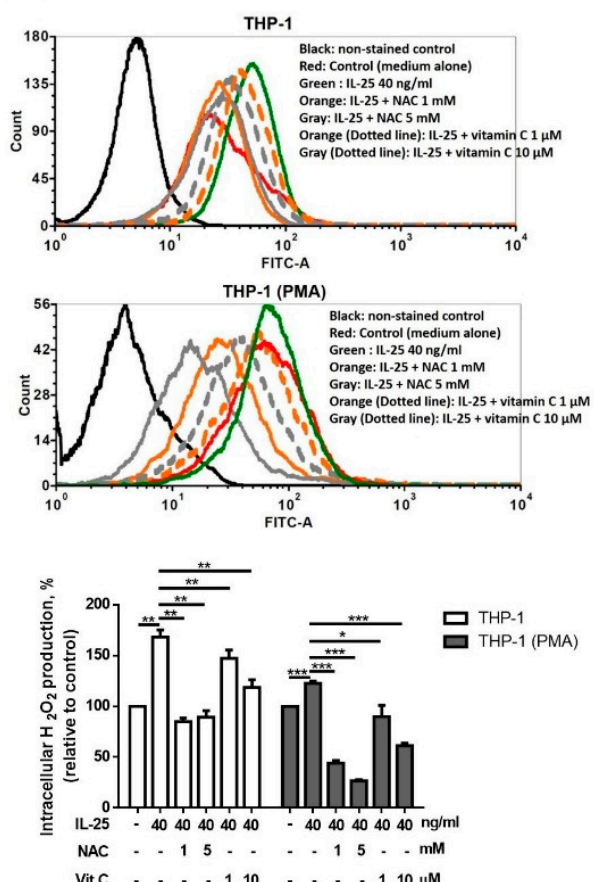

Figure 1. IL-25 alone could induce intracellular ROS level and suppressed by NAC, vitamin C, antimycin A and MitoTEMPO. (A-E), representative histograms of cell counts (counts) vs. DCF fluorescence (FITC-A) (above), and the mean fluorescence intensity (MFI) of DCF is expressed as percentage relative to control cells (below). THP-1 cells (white bar) and THP-1 derived macrophages (gray bar) treated with medium alone or IL-25 (2, 10 and $40 \mathrm{ng} / \mathrm{mL})$ was for $0.5 \mathrm{~h} \mathrm{(A)} \mathrm{or} 2 \mathrm{~h}($ B) . (C) Cells were treated with medium alone or IL-25 $(40 \mathrm{ng} / \mathrm{mL})$ present pretreatment with NAC (1 and $5 \mathrm{mM})$ or vitamin $\mathrm{C}(1$ and $10 \mu \mathrm{M})$ for $0.5 \mathrm{~h}$. After pretreatment with solvent control $(0.1 \% \mathrm{DMSO})$ or antimycin A (0.1 and $0.5 \mu \mathrm{g} / \mathrm{mL})(\mathbf{D})$, or medium alone or MitoTEMPO $(10$ and $100 \mu \mathrm{M})(\mathrm{E})$ for $0.5 \mathrm{~h}$ followed by IL-25 treatment, the ROS level was measurement. ${ }^{*} p<0.05$, ${ }^{* *} p<0.01$, and ${ }^{* * *} p<0.001$; $\mathrm{n}=3$, means $\pm S D$. 


\subsection{IL-25 Increased Mitochondrial Complex I and II/III Activity}

IL-25 could induce ROS production in THP-1 cells and THP-1 derived macrophages, and mitochondria are the major source of ROS production. Therefore, we further measured the mitochondrial complex activity of THP-1 cells and THP-1 derived macrophages after IL-25 stimulation. As shown in Figure 2A, the activity of mitochondrial complex I in THP-1 cells and THP-1 derived macrophages was significantly increased by IL-25 stimulation in a dose-dependent manner. In addition, we also observed that IL-25-induced mitochondrial complex II/III activity of THP-1 cells and THP-1 derived macrophages was significantly increased (Figure 2B).

(A)

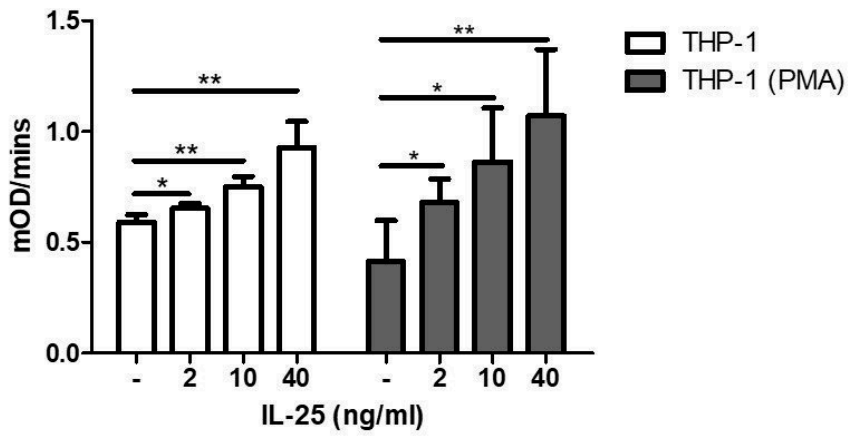

(B)

\section{Complex II/III activity}

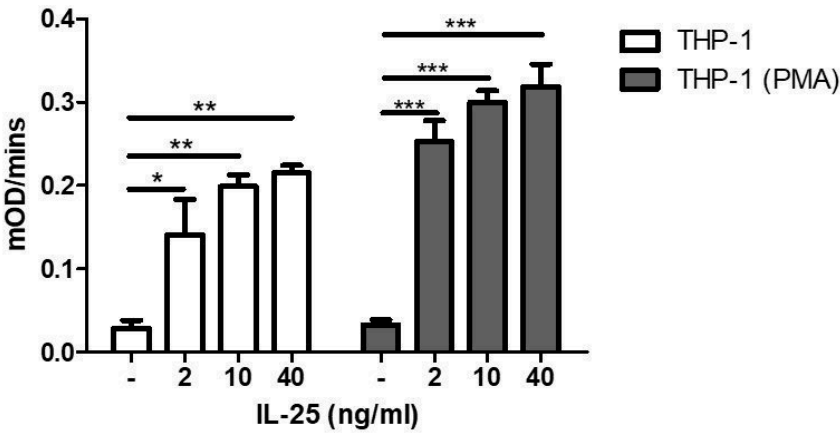

Figure 2. IL-25 increased mitochondria complex activity in THP-1 cells and THP-1 derived macrophages. THP-1 cells (white bar) and THP-1 derived macrophages (gray bar) were treated with medium alone or IL-25 $(2,10$, and $40 \mathrm{ng} / \mathrm{mL})$ for $2 \mathrm{~h}$. The activity of complex I (A) and complex II/III (B) by IL-25 treatment were determined. ${ }^{*} p<0.05,{ }^{* *} p<0.01$, and ${ }^{* * *} p<0.001 ; \mathrm{n}=4$, means $\pm \mathrm{SD}$.

\subsection{IL-25 Induced AMPK Activation and Mitophagy-Related Proteins Expression}

IL-25 significantly induces ROS production, and ROS plays an important role in mitophagy. Mitophagy is essential for cellular energy and maintaining redox homeostasis and important for mitochondrial quality control [14]. Previous research observed that a lack of skeletal muscle AMPK resulted in the failed induction of mitophagy-related signaling and development of apparent mitochondrial abnormalities. [15]. Thus, we further examined whether IL-25 can also induce AMPK activation and mitophagy-related proteins expression in THP-1 cells and THP-1 derived macrophages. Western blot analysis showed that IL-25 significantly increased the levels of p-AMPK at $12 \mathrm{~h}$ in THP-1 cells but not in THP-1 derived macrophages. However, the best time point of p-AMPK activation is $8 \mathrm{~h}$ (Figure 3A). In THP-1 cells and THP-1 derived macrophages, the expression of p-AMPK and the mitophagy-related proteins PINK1, p-Parkin, and LC3 was significantly increased by IL-25 treatment with a dose-dependent manner at $8 \mathrm{~h}$ (Figure 3B-E). Costaining of mitochondria and LC3 was used to monitor IL-25-induced mitophagy. As shown in Figure 3F, mitochondria and LC3 were colocalized, and their colocalization increased in response to $10 \mathrm{ng} / \mathrm{mL}$ IL-25 stimulation in THP-1 cells and THP-1 derived macrophages, as shown by confocal laser microscopy analysis. 
(A)

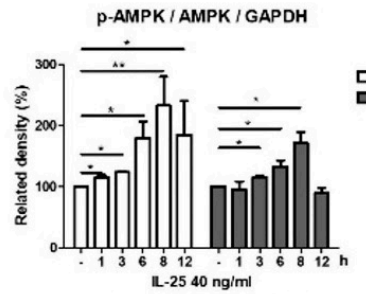

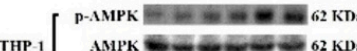

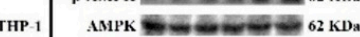

GAPD $=-0-0-137 \mathrm{KD}$

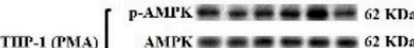
GAPDI $=0$ $11 \mathrm{~L}-2540 \mathrm{ng} / \mathrm{ml}-\begin{array}{lllllll}1 & 3 & 6 & 8 & 12 & \text { b }\end{array}$

(D)

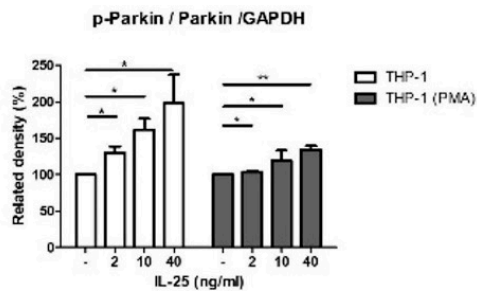

[ p-parkin $---52 \mathrm{kD}$.

THP-1 purkin $=-52 \mathrm{KDa}$ GAPH 0 $\left[{ }^{p-p u r k i n ~}=-252 \mathrm{k \omega}\right.$. parkin $-=-52 \mathrm{KD}$ GAPDH

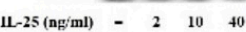

(F)

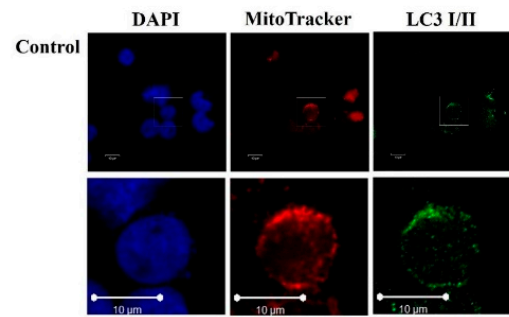

IL-25 $10 \mathrm{ng} / \mathrm{ml}$
(B)

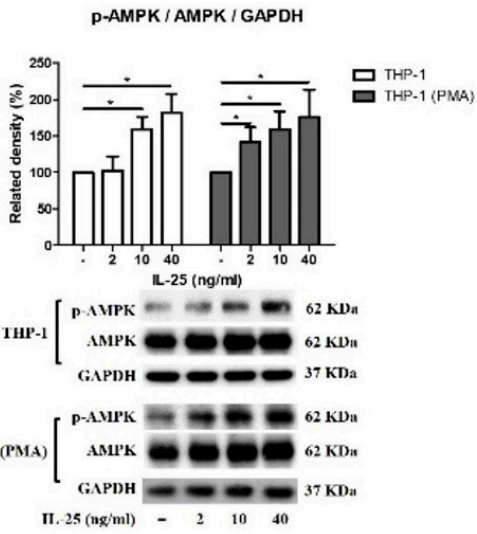

(E)

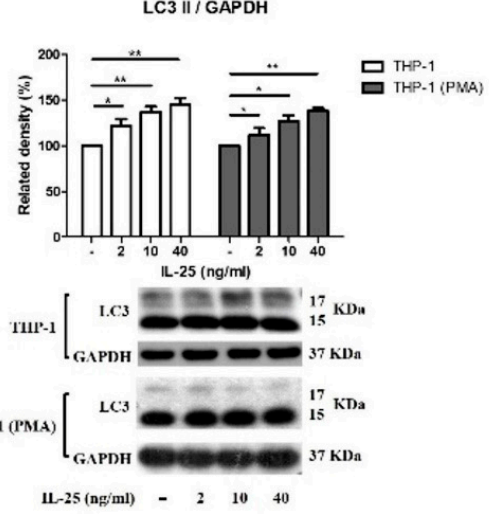

(C)

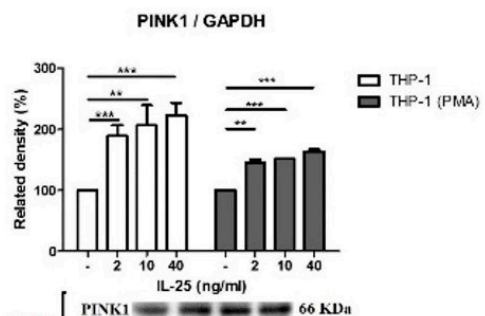

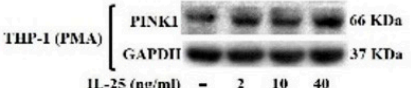
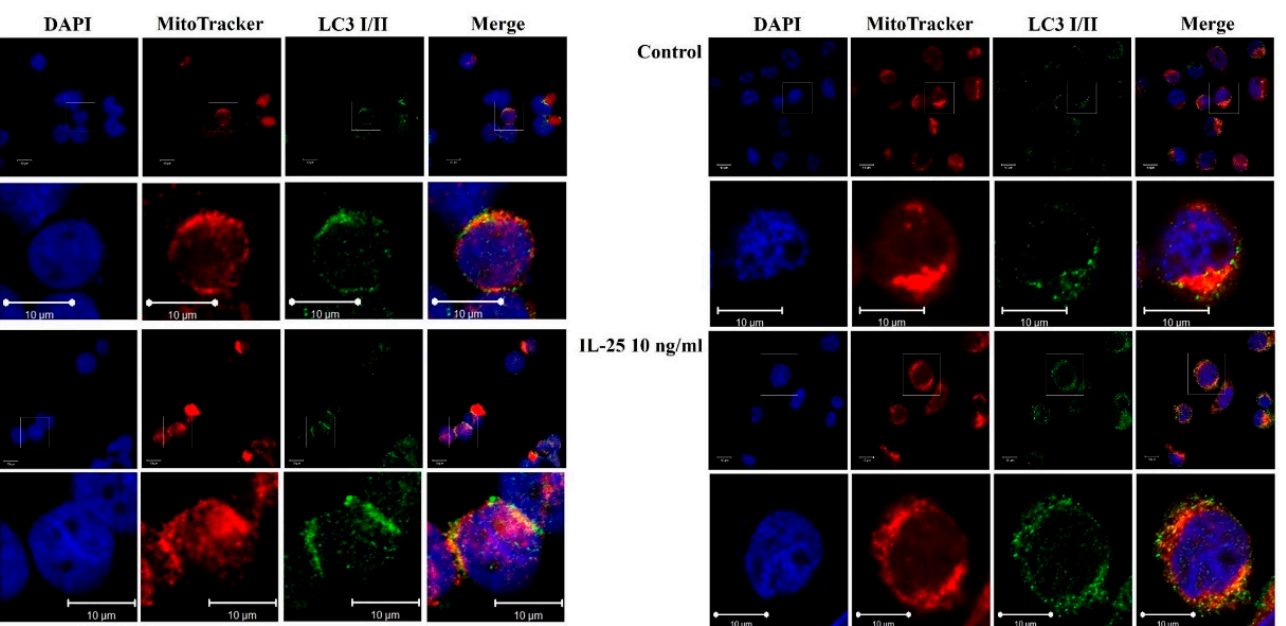

Figure 3. IL-25-induced AMPK activation and mitophagy-related proteins expression in THP-1 cells and THP-1 derived macrophages. (A) The time course of p-AMPK expression was determined at 1 , $3,6,8$, and $12 \mathrm{~h}$ with IL-25 (40 $\mathrm{ng} / \mathrm{mL})$ or medium alone treatment in THP-1 cells (white bar) and THP-1 derived macrophages (gray bar). Data present the means \pm SD of 3 independent experiments. The expression of p-AMPK (B), PINK1 (C), p-parkin (D), and LC3 (E) by medium alone or IL-25 $(2,10$, and $40 \mathrm{ng} / \mathrm{mL}$ ) treatment in THP-1 cells (white bar) and THP-1 derived macrophages (gray bar) at $8 \mathrm{~h}$ time point were determined by western blot. ${ }^{*} p<0.05,{ }^{* *} p<0.01$, and ${ }^{* * *} p<0.001$; $\mathrm{n}=3$, means \pm SD. The confocal data show the nuclear (DAPI), mitochondria (MitoTracker), and LC3 I/II fluorescence images by medium alone or IL-25 $(10 \mathrm{ng} / \mathrm{mL})$ treatment $(\mathbf{F})$. Scale bars $=10 \mu \mathrm{m}$ in pictures. 


\subsection{IL-25 Induced Mitophagy through the ROS-AMPK Pathway}

AMPK has been suggested to be a redox-sensing protein that mediates mitophagyrelated signaling.

Therefore, we further examined the relationship between ROS and AMPK activation after IL-25 stimulation. In this study, pretreated AMPKi caused enhanced ROS production in IL-25-treated THP-1 cells and THP-1 derived macrophages (Figure 4A). Moreover, we also used NAC, rotenone, and antimycin A to observe whether IL-25 induced AMPK activation. The IL-25-increased p-AMPK expression was suppressed by NAC (Figure 4B). The IL-25-increased p-AMPK was also significantly suppressed by rotenone and antimycin A (Figure 4C). Thus, it suggested that complexes I and III produced mitochondrial ROS involved in AMPK activation. We further used the AMPKi to examine whether IL-25 induced mitophagy-related proteins expression in THP-1 cells and THP-1 derived macrophages. The Western blot data showed that IL-25-induced PINK expression was suppressed by treatment with the AMPKi (Figure 4D). In addition, the IL-25-induced increases in the levels of p-Parkin and LC3 were similarly suppressed by treatment with the AMPKi (Figure 4E,F). The data supported that L-25-induced mitophagy was through increased ROS production and AMPK activation. We further used mdivi-1 to investigate ROS production and mitophagy-related proteins expression on IL- 25 treatment in THP-1 cells and THP- 1 derived macrophages. As shown in Figure 5A, IL-25-induced ROS production was inhibited by mdivi-1 pretreatment. The IL-25-induced mitophagy-related protein (PINK1, p-Parkin, and LC3) expression decreased by mdivi-1 (Figure 5B-D). Furthermore, we also knock down PINK1 to examine whether IL-25 induced ROS production and mitophagy-related proteins expression. The knockdown efficiency of PINK1 is shown in Figure 5E. We selected TRCN0000007097 and TRCN0000199446 shRNA to investigate further experiments. After PINK1 knockdown, the ROS production of THP-1 cells and THP-1 derived macrophages was increased without IL-25 stimulation. Moreover, ROS production was not enhanced by IL-25 treatment (Figure 5F). The data supported that PINK1 was involved in IL-25-induced ROS production. We further examine whether IL-25 induced mitophagy-related protein expression in THP-1 cells and THP-1 derived macrophages. As shown in Figure 5G, the result indicated the successful knockdown PINK1. After PINK1 knockdown, the PINK1 downstream proteins (p-Parkin and LC3) were not affected by IL-25 treatment (Figure 5H,I). Together, these results suggested that IL-25-induced mitophagy via PINK1-Parkin pathway through ROS and AMPK activation.

\subsection{IL-25-Induced Mitophagy Was Associated with an M2 Macrophage Polarization Shift}

To evaluate the functional outcomes of IL-25-induced mitophagy in THP-1 cells, we examined the effects of IL- 25 on macrophage polarization. IL-25 stimulation significantly decreased the release of the M1-related cytokines CXCL-10 and TNF- $\alpha$ in THP-1-derived M1 cells but did not show the effect from THP-1 cells and THP-1-derived macrophage. (Figure 6A,B). In contrast, the M2-related cytokine CCL-22 was significantly increased in THP-1-derived M2 cells but not shown in THP-1 cells and THP-1- derived macrophage (Figure 6C). These results suggested that IL-25 promoted macrophage polarization toward the M2 phenotype. Furthermore, IL-25-induced production of M2-related cytokines was suppressed by treatment with the Mdivi-1 or genetic knockdown of PINK1 (Figure 6D,E), indicating that the IL-25-induced M2 macrophage polarization shift was mediated by mitophagy. 
(A)
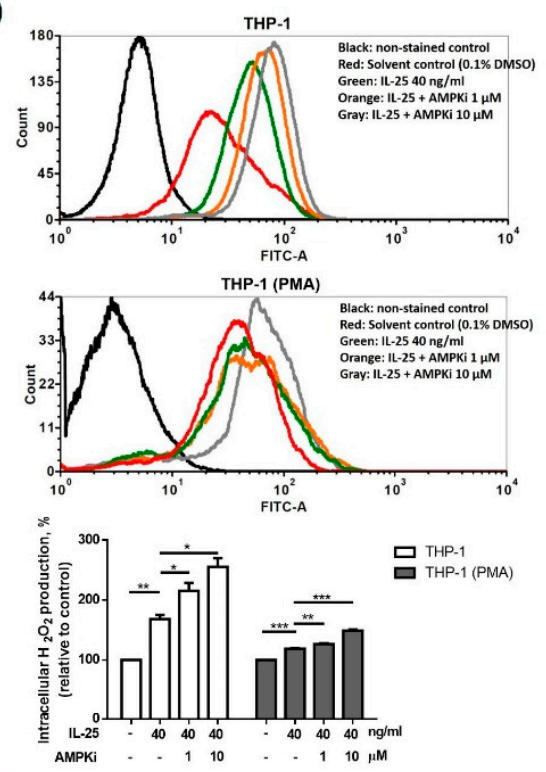

(D)
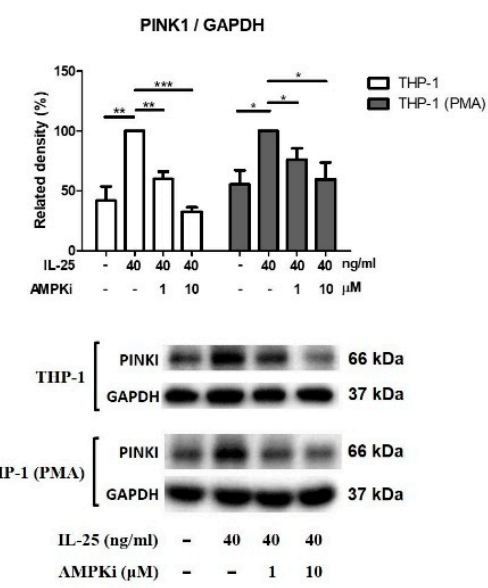

(B)

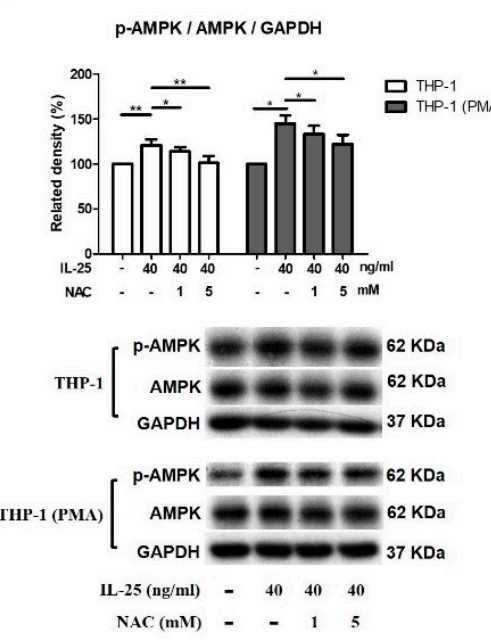

(E)

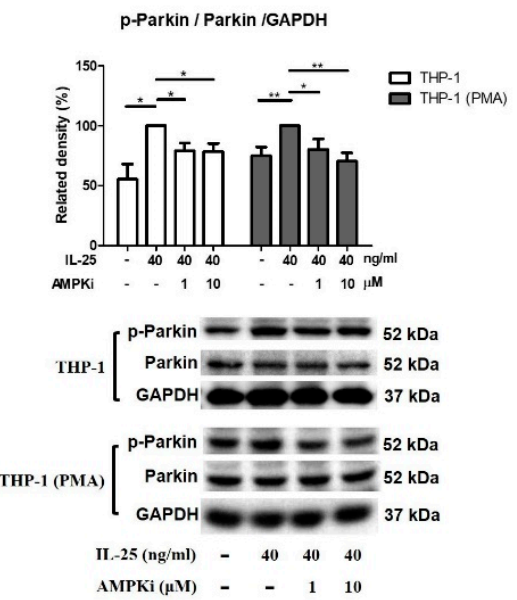

(C)
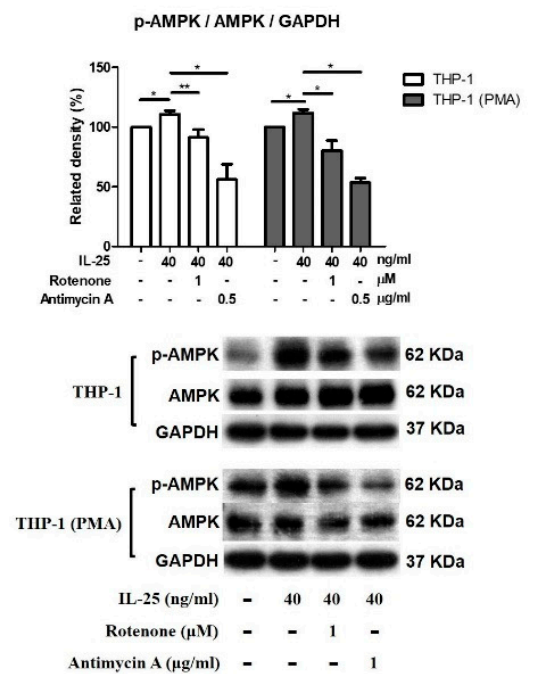

(F)

LC3 II / GAPDH

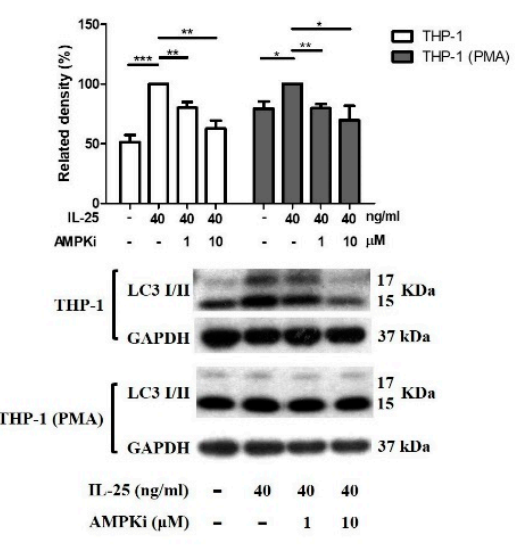

Figure 4. IL-25-induced mitophagy-related proteins expression via ROS-AMPK pathway in THP-1 cells and THP-1 derived macrophages. (A) representative histograms of cell counts (counts) vs. DCF fluorescence (FITC-A) (above), and the mean fluorescence intensity (MFI) of DCF expressed as a percentage relative to control cells (below). (A) After pretreatment with solvent control (0.1\% DMSO) or AMPKi (1 and $10 \mu \mathrm{M})$ for $0.5 \mathrm{~h}$, the IL-25-induced ROS production in THP-1 cells (white bar) and THP-1 derived macrophages (gray bar) were measured. $n=3$, means \pm SD. (B) After medium alone or NAC ( 1 and $5 \mathrm{mM}$ ) pretreatment for $0.5 \mathrm{~h}$ followed by IL-25 treatment for $8 \mathrm{~h}$, IL-25-induced p-AMPK expression in THP-1 cells (white bar) and THP-1 derived macrophages (gray bar) were determined. $n=4$, means \pm SD. (C) After pretreatment with solvent control ( $0.1 \% \mathrm{DMSO})$, rotenone $(1 \mu \mathrm{M})$ or antimycin A $(0.5 \mu \mathrm{g} / \mathrm{mL})$ for $0.5 \mathrm{~h}$ followed by IL-25 treatment for $8 \mathrm{~h}$, IL-25-induced p-AMPK expression in THP-1 cells (white bar) and THP-1 derived macrophages (gray bar) were deter-mined. $\mathrm{n}=3$, means \pm SD. After pretreatment with solvent control (0.1\% DMSO) or AMPKi (1 and $10 \mu \mathrm{M}$ ) for $0.5 \mathrm{~h}$ followed by IL-25 treatment for $8 \mathrm{~h}$, the PINK1 (D), p-Parkin (E), and LC3 I/II (F) protein levels were determined by western blot. $\mathrm{n}=3$, means $\pm \mathrm{SD}$. ${ }^{*} p<0.05,{ }^{* *} p<0.01$, and *** $p<0.001$. 
(A)
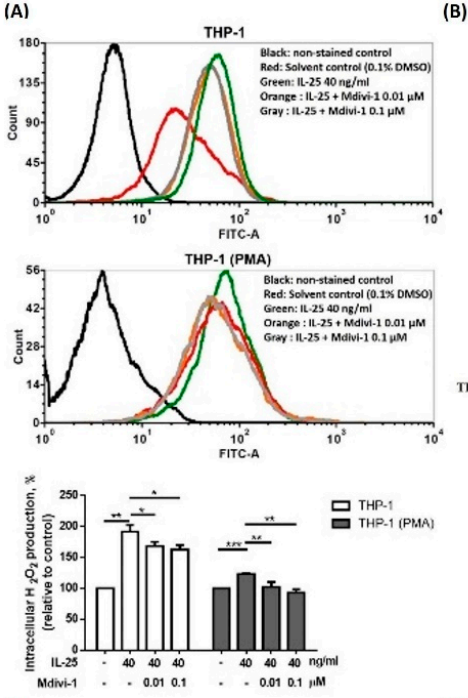

(D)
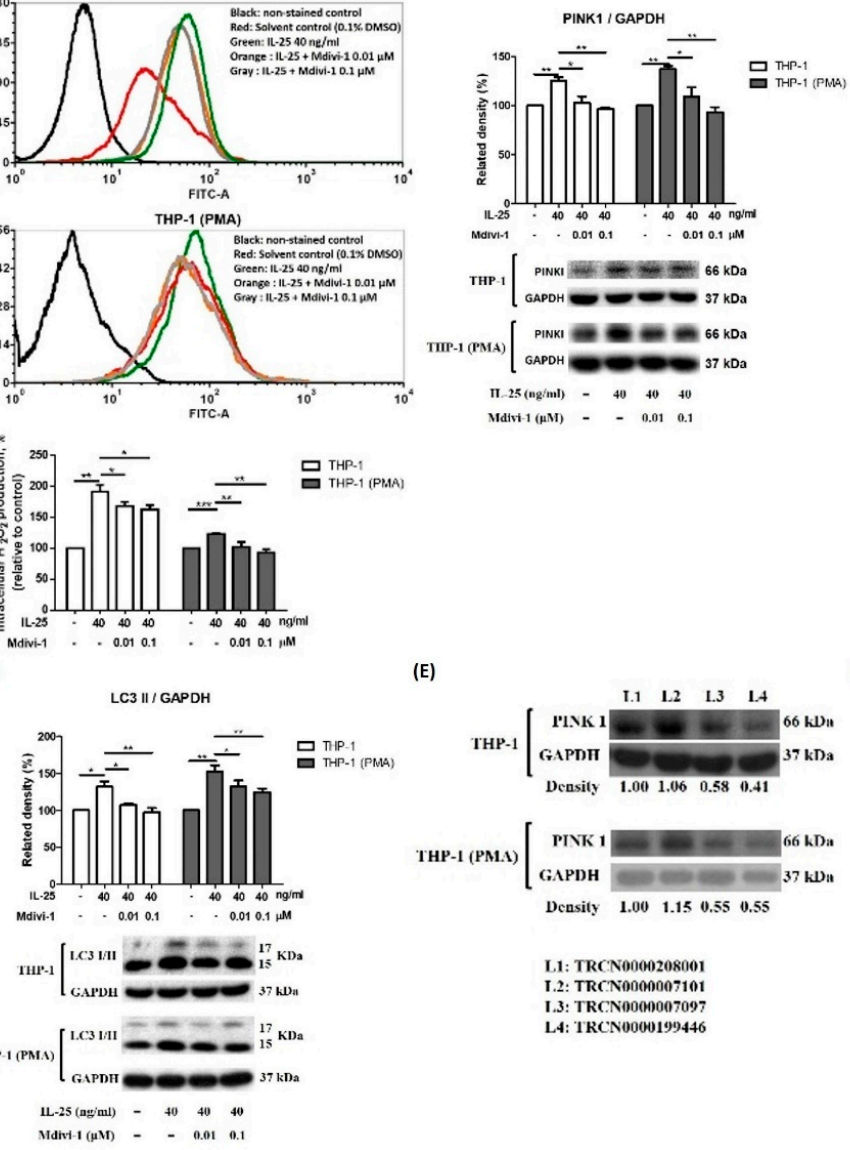

TIIP-1 (PMA) $\left[{ }_{\text {PINKI }}=\mathbf{0}=66 \mathrm{kDa}\right.$

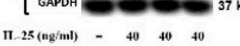

(4)

1. TRCN0000208001

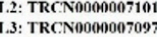
L4: TRCN0000199446
(C)

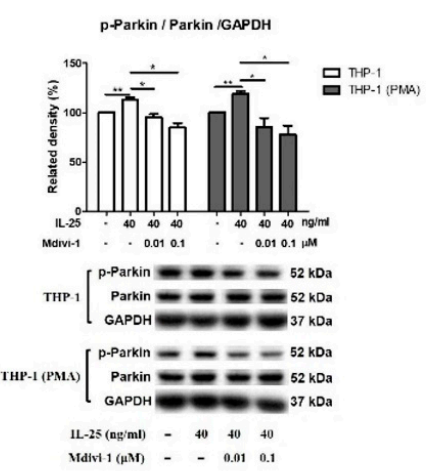

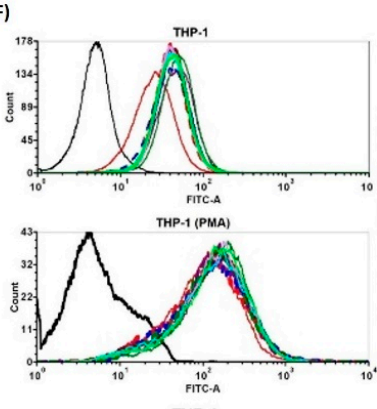

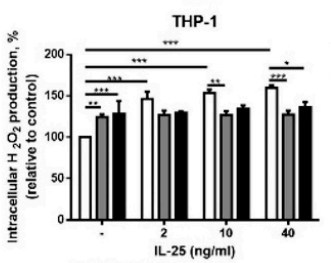

Nan-largeting control

ShPINK1 (TRCN0000007097)
Dotted line: non-stained control
Red: Control (medium alone) Red: Control (medium al
Blue: Il-25 $2 \mathrm{ng} / \mathrm{ml}$ Blue: $11-252 \mathrm{ng} / \mathrm{ml}$
Purple: $11-2510 \mathrm{ng} / \mathrm{m} /$
Green: $11-2540 \mathrm{ng} / \mathrm{m} /$ Green: II-25 $40 \mathrm{ng} / \mathrm{ml}$
Red (Dotted line): : ShPINK1 (7097) control

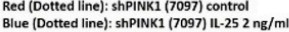

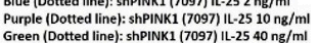
Green (Dotted line): shPINK1 (70977) IL-25 40 nefl
Dark red: shPPINK1 (199446) control Turquoise : ShPPINK1 (199946) II-25 2 $2 \mathrm{gg} / \mathrm{ml}$ Lavender: shPINK1 (199446) II-25 $10 \mathrm{ng} / \mathrm{ml}$
Bright Green : shPINK1 (199446) IL-25 40 ng/ml
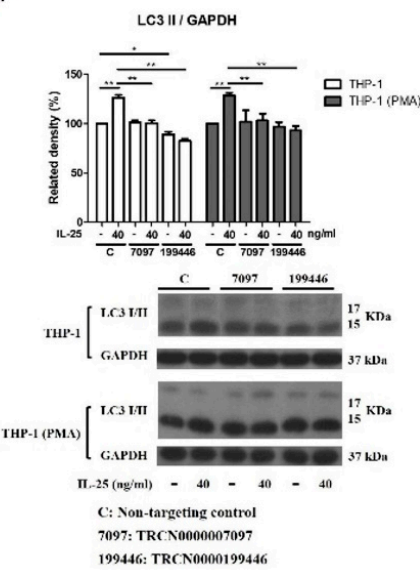

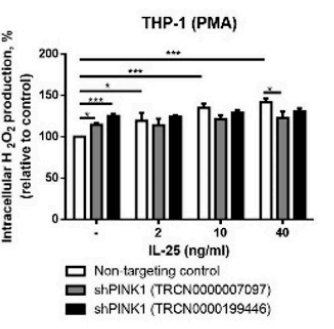

믐 stPINK1 (TRCN0000007097)
(G)
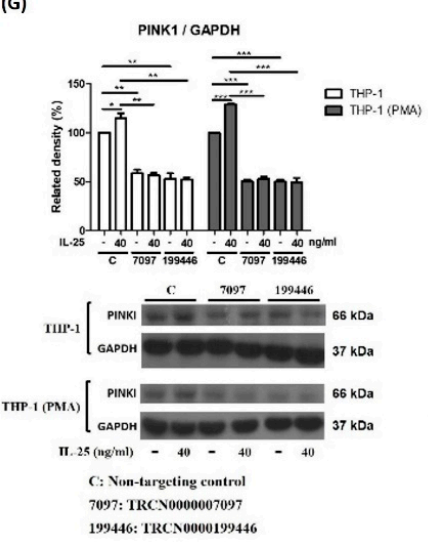

(H)

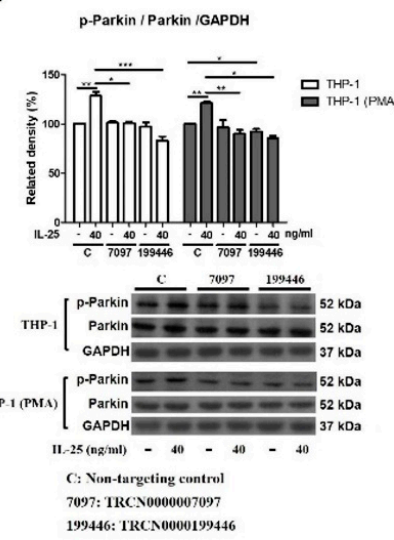

Figure 5. IL-25-induced mitophagy via PINK1-Parkin pathway in THP-1 cells and THP-1 derived macrophages. (A,F) representative histograms of cell counts (counts) vs. DCF fluorescence (FITC-A) (above), and the mean fluorescence intensity (MFI) of DCF expressed as a percentage relative to control cells (below). (A) After pretreat-ment with solvent control (0.1\% DMSO) or mdivi-1 for $0.5 \mathrm{~h}$, the IL-25-induced ROS production in THP-1 cells (white bar) and THP-1 derived macrophages (gray bar) were measured by flow cytometry. $n=3$, means \pm SD. After pretreatment with solvent control 
(0.1\% DMSO) or mdivi-1 for $0.5 \mathrm{~h}$ followed by IL-25 treatment for $8 \mathrm{~h}$, the PINK1 (B), p-Parkin (C), and LC3 I/II (D) protein levels of THP-1 cells (white bar) and THP-1 derived macrophages (gray bar) were determined. $n=3$, means \pm SD. (E) After transduction with shRNA lentiviral particles and puromycin selection for $3 \mathrm{~d}$, the PINK1 expression was determined. (F) After PINK1 knockdown by TRCN0000007097 or TRCN0000199446 shRNA, the ROS level by medium alone or IL-25 (2, 10, and $40 \mathrm{ng} / \mathrm{mL}$ ) treatment in THP-1 cells at $2 \mathrm{~h}$ was measured. $\mathrm{n}=3$, means \pm SD. The expression of PINK1 (G), p-Parkin (H), LC3 I/II (I) by medium alone or IL-25 (40 ng/mL) treatment following transduction with PINK1 shRNA or non-targeting control were determined. $\mathrm{n}=3$, means \pm SD. ${ }^{*} p<0.05,{ }^{* *} p<0.01$, and ${ }^{* *} p<0.001$.

(A)

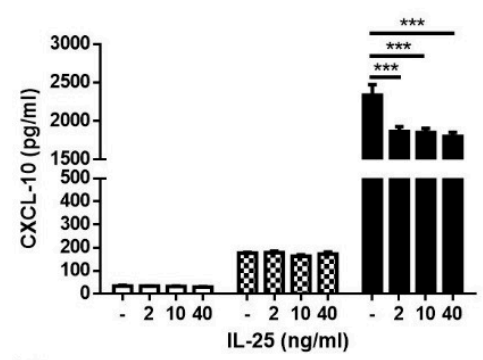

(C)

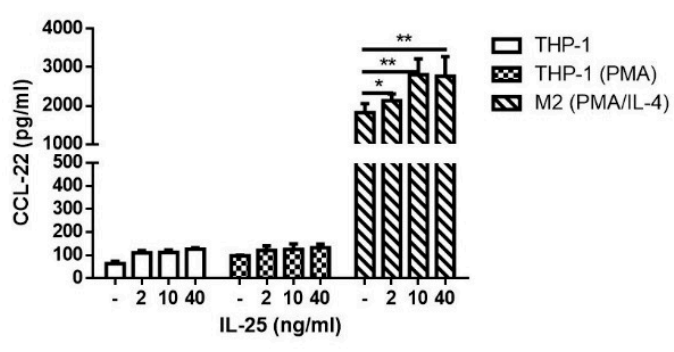

(B)

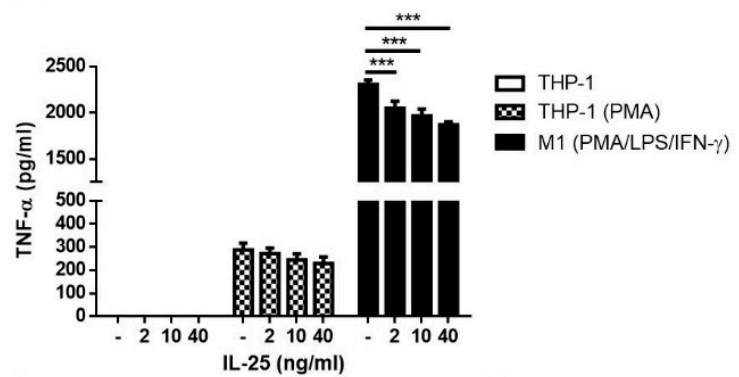

(D)

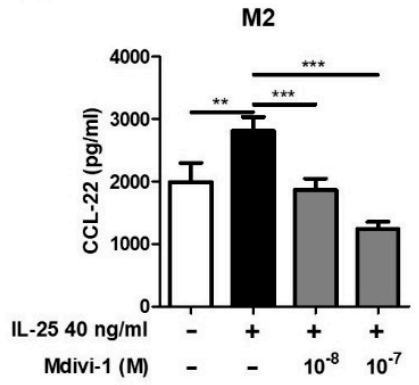

(E)

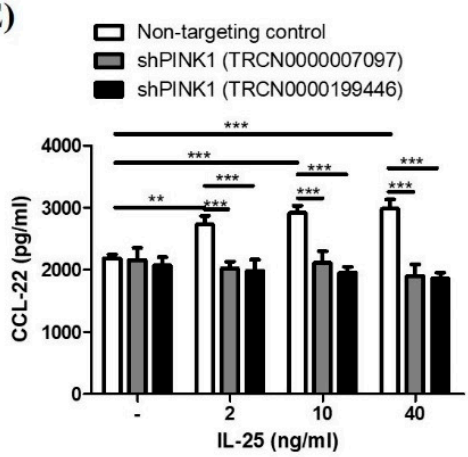

Figure 6. IL-25-induced mitophagy was associated with a shift in M2 macrophage polarization. THP1 cells were pretreated with medium alone or IL-25 $(2,10$, and $40 \mathrm{ng} / \mathrm{mL})$ for $2 \mathrm{~h}$ and differentiated to THP-1 derived macrophages (PMA), M1 (PMA/LPS/IFN- $\gamma$ ), or M2 (PMA/IL-4) macrophages. The concentration of M1-related chemokine CXCL-10 (A) and cy-tokine TNF- $\alpha$ (B) and M2-related cytokine CCL-22 (C) were measured. After pretreatment with solvent control (0.1\% DMSO) or mdivi-1 (D), or knockdown PINK1 (E), the IL-25-induced CCL-22 production was assessed. ${ }^{*} p<0.05$, ** $p<0.01$, and ${ }^{* * *} p<0.001 ; \mathrm{n}=3$, means $\pm \mathrm{SD}$.

\section{Discussion}

Asthma is a chronic disease characterized by reversible airflow limitation, bronchial constriction, and inflammatory immune response. Epithelial cells are the first line against allergen exposures and environmental stimuli via the expression of many cytokines and chemokines such as IL-25. IL-25 induces proallergic chemokine production, an increase in goblet cells, and mucus secretion in airway epithelium, resulting in epithelial hyperplasia and airway hyperreactivity [16]. IL-25 also mediated immune cells, such as dendritic cells (DCs) and macrophages, to activate a type 2 immune response. A previous study demonstrated the IL-25 receptor IL-17RB expression of memory Th2 cells was significantly increased by coculture with TSLP-conditioned DCs (CD11c $\mathrm{c}^{+}$DCs cultured with $15 \mathrm{ng} / \mathrm{mL}$ of TSLP for $24 \mathrm{~h}$, then washout) and enhanced proliferation of memory Th2 cells by IL-25 stimulation [17]. In a human study, the IL-17RB was upregulated in myeloid DCs (mDCs) and plasmacytoid DCs via allergen challenge and coincident with increases in airway eosinophils. Thus, it suggested that IL-25 may activate mDCs to initiate type 2 immune responses [18]. In the airway, macrophages are the most abundant immune cells and 
interact with epithelial cells to maintain immune tolerance and tissue repair [19]. However, few studies have investigated epithelial-macrophage crosstalk, even the role of IL-25 in macrophages. ROS are involved in many cell physiological processes. However, they are also implicated in the pathogenesis of tissue injury. In the asthmatic airway, inflammatory cells are recruited and produce various highly reactive ROS to promote tissue damage and chronic airway inflammation [20,21]. The main sources of oxidants in the bronchial airways are environmental pollution, e.g., particulate matter (PM) and endogenously produced oxidants due to local inflammation [22]. IL-25, also named IL-17E, is a distinct member of the IL-17 cytokine family, whose members can promote and augment Th2 cells. Clinical studies have indicated that IL-25 production is associated with eosinophilic inflammation and mucus hypersecretion, and with AHR and airway remodeling [13]. In the airway, the direct targets of inhaled PM are epithelial cells and antigen-presenting dendritic cells (DCs). Several cytokines are mediated by epithelial cells, including IL-25, which promoting the DC response to allergens and PM. PM components are also redox-active and can induce cellular oxidative stress and injuries, including inflammation and cell death [23]. Therefore, recent studies have suggested that a novel method to monitor disease severity is to assess oxidative stress byproducts. In addition, a potential treatment strategy was used in asthmatic patients by reducing ROS levels to decrease airway inflammation, which is produced by oxidative stress [24]. The present study results showed that IL-25 could increase ROS production in monocytes, suggesting that IL-25 may be important not only in the induction of Th2 cytokine production, but also as a potent source of oxidative stress. In our data, the intracellular ROS level was not obviously different between THP-1 and THP-1 (PMA) cells. Both monocytes and macrophages could produce ROS. However, macrophages have more oxidative burst activity and reactive oxygen species (ROS) production due to their capacity to phagocytize foreign materials, especially M1 macrophage [25]. Our experiment condition only added PMA, not combined IFN- $\gamma$ and LPS, and differentiated $24 \mathrm{~h}$, not three days or more. Our PMA-primed THP-1 cells are not mature M1 macrophages. The production and release of epithelial cytokines, including IL-33, IL-25, and TSLP, lead to the recruitment and activation of ILC2, which secretes mediators, such as IL-5 and IL-13, that augment allergic inflammation in the pathogenesis of virus-induced asthma exacerbation. The most common cause of virus-induced asthma exacerbation and accounting for much of the morbidity burden of asthma is rhinovirus (RV) infection. RV-infected asthmatic bronchial epithelial cells displayed an enhanced intrinsic capacity for IL-25 expression, which could contribute to asthma exacerbation [26]. Early-life human RV infection has been associated with asthma development in high-risk infants and children. An RV-induced asthma animal model revealed an increased intrinsic capacity for type 2 cytokines in the lung, and IL25 is a key mediator of RV-induced exacerbation of pulmonary inflammation, especially in early life [27]. It has been reported that the levels of IL-25 and its receptor IL-17RB were increased in respiratory syncytial virus (RSV) infection, and RSV-associated AHR and type 2 cytokine production were reduced because of IL-25 blockade by neutralizing antibodies. An IL-17RB ${ }^{-/-}$mouse study demonstrated that decreases in Th2 cytokines and increases in Th17 cytokines reduced inflammation in mice with RSV-induced asthma exacerbation [28]. Therefore, the RSV-induced inflammatory response was regulated by IL-25, which might reduce the severity of RSV-associated pulmonary inflammation and viral-induced asthma exacerbation via IL-25 blockade. These environmental factors increase the levels of cellular ROS and induce mitochondrial dysfunction in the airway epithelium of asthmatic patients [29]. The imbalance in mitochondrial ROS production, which consists principally of the generation of superoxide and hydrogen peroxide, has been implicated in dysfunction in the electron transport chain (ETC), resulting in altered ROS production. However, the response of IL-25, a Th2-like cytokine, to the ETC has not been thoroughly assessed. Complex I and III redox centers in the respiratory chain of mitochondria have been implicated as the major sites of mitochondrial ROS production, and complex II is also capable of producing ROS [30,31]. In the present study, our results showed that the complex I and II/III activities after $2 \mathrm{~h}$ of stimulation by IL- 25 were significantly increased compared 
to the control group. Thus, IL-25 may increase ROS generation by expanding complex I and II/III activity. However, an increase in the activity of the complexes is not necessarily related to a rise in ROS levels. Previous research showed increased ROS production but reduced complex I activity in mitochondria from reperfused rat heart [32]. Other research indicated that mitochondria's complex II or III activity increase could follow increased complex II or III-associated ROS production [33,34]. The present study showed IL-25 could increase mitochondrial complex I and II/III activity and ROS production.

Mitochondria play an important metabolic role, cause cellular damage via oxidative phosphorylation byproducts, and trigger apoptosis via cytochrome c release from damaged mitochondria and the activation of cell death pathways [35]. Mitophagy is the selective degradation of mitochondria by autophagy and occurs in defective mitochondria following damage or stress. The pathways for generating mitophagy have been identified and are primarily mediated by PINK1 and the E3 ubiquitin ligase Parkin [36,37]. PINK1 is constitutively expressed and imported to healthy mitochondria via the translocase TIM/TOM complex to the inner membrane and is cleaved by presenilin-associated rhomboid-like protein (PARL). Parkin is kept in a 'closed' confirmation through multiple intramolecular interactions that keep the enzyme in an autoinhibited state. In damaged mitochondria, activated PINK1 accumulates on the mitochondrial surface upon loss of mitochondrial membrane potential [38]. A previous study indicated that PINK1 could be autophosphorylated by PINK kinase. Accumulated PINK1 activates Parkin by phosphorylating the Ser-65 residue of Parkin and recruiting Parkin to mitochondria to trigger mitophagy [39]. Recently, the mechanism of mitochondrial dysfunction has been established to be linked with the pathogenesis of chronic respiratory diseases by playing a causative role in the structural remodeling of the lung. TGF- $\beta 1$, a key cytokine for asthma-related pulmonary fibrosis, could induce lung epithelial cell mitochondrial ROS and stabilize the key mitophagy initiating protein PINK1. PINK1 ameliorates epithelial cell death and is associated with fibrogenesis [40]. Increased pulmonary expression of IL-25 is found in idiopathic pulmonary fibrosis, and a population of ILC2s is also observed in the lungs of idiopathic pulmonary fibrosis patients [6]. In the present study, IL-25 induced ROS production and subsequently increased mitophagy-related PINK1, Parkin, and LC3 expression. These results may explain the association between IL-25 and idiopathic pulmonary fibrosis.

AMPK, a trimeric complex including the $\alpha, \beta$, and $\gamma$ subunits, is the main cellular energy sensor that restores energy homeostasis and coordinates metabolism. Full activation of AMPK occurs through threonine phosphorylation in the activation loop of the kinase domain, which is situated at the $\mathrm{N}$-terminus of the $\alpha$ subunit. Increasing evidence suggests that AMPK might also be a redox-sensing protein [41]. Mitochondrial ROS activates AMPK under both resting and stress conditions [42]. Mitochondrial complexes I and III are major sources of mitochondrial ROS. In 2004, Zou MH. et al. reported that mitochondrial $\mathrm{O}_{2}{ }^{\bullet-}$ was increased by rotenone treatment and led to increased phosphorylation of AMPK [43]. In a previous study, Rabinovitch et al. also indicated that "mitochondrial ROS are a noncanonical activator of AMPK" [44]. It seems that mitochondrial ROS produced by complexes I and III are involved in AMPK activation. A previous study observed that a nonprescription oral drug, berberine, which is adjunctively for metabolic disorders, increased peroxynitrite, a potent oxidant producer, and phospho-AMPK expression. Berberine-induced phosphoAMPK expression was attenuated by antioxidant NAC pretreatment [45]. This study also showed that phospho-AMPK expression was increased by IL-25 stimulation and was decreased by NAC pretreatment. AMPK regulates a variety of metabolic processes, including mitochondrial biology and homeostasis. In mitochondrial homeostasis, AMPK can directly phosphorylate mitochondrial fission factor (MFF) to regulate mitochondrial fission, which is required for mitophagy, and activate ULK1, the upstream kinase in autophagy and mitophagy [46]. In the present study, we used an AMPK inhibitor to investigate the effect of AMPK on regulating mitophagy-related proteins expression. Our results showed that IL-25-increased mitophagy-related proteins, as PINK1, p-Parkin, and LC3 expression were attenuated by AMPK inhibitor pretreatment. Mitochondrial biogenesis has been indicated 
to be regulated by peroxisome proliferator-activated receptor)- $\gamma$ coactivator- $1 \alpha$ (PGC-1 $\alpha$ ). PGC-1 $\alpha$ has been suggested to increase the expression of key mitochondrial enzymes, such as ATP synthetase ( $\beta$-subunit) and cytochrome c oxidase (COX) subunits (COX II and COX IV). An animal model showed a strong positive correlation between PGC-1 $\alpha$ protein expression and COX activity [47]. More signalling pathways were demonstrated leading to the PGC- $1 \alpha$ transcription cascade activation, such as p38 mitogen-activated protein kinases (p38 MAPK) and AMPK [48]. IL-25 has been shown to be mediated by their receptors IL-17RA and IL-17RB and to activate several downstream signalling cascades, including MAPKs, nuclear factor kappa B (NF-kB), and Janus kinase/signal transducer and activator of transcription (JAK/STAT) [16]. We found IL-25 could induce AMPK activation by ROS and mediated mitophagy. Taken together, it seems that IL-25 stimulated mitochondrial activity through AMPK and subsequent mitophagy. All our data also suggested that epithelium-derived IL-25 may not only induce Th2-related cytokine expression, but also ROS production, and subsequently influence cellular oxidative stress and the normal redox state and regulate mitochondrial quantity in monocytes (Figure 7). The present study indicated that IL-25 could increase intracellular ROS level and mitochondrial complex I and II/III activity and induce mitophagy through AMPK activation and PINK1-Parkin pathway in human monocyte cell line THP-1 and THP-1 (PMA). We further investigated whether IL-25 could enhance M2 chemokine CCL-22 production in M2 macrophage and suppress by mitophagy inhibitor or PINK1 knockdown. In addition, a previous study observed that mitochondrial respiratory capacity, Arg-1 (one of M2 marker), and Th2 cytokines such as IL-4, IL-5, and IL-13 were increased in IL-25-induced bone marrow-derived macrophages. Furthermore, in IL-25-treated high-fat diet-fed mice, the ARG1 and F4/80 double-positive cells accumulated in both the liver and epididymal white adipose tissue [49]. Taken together, it seems that IL-25 could train macrophages to skew towards M2 phenotype through mitophagy and induce Th2-type immune responses. Understanding the mechanisms of IL-25-induced ROS production and mitophagy, which are important factors for the pathogenesis of asthma, allergies, and other airway diseases, can help us identify viable points for therapeutic intervention in respiratory diseases and for future avenues of research in this rapidly evolving field.

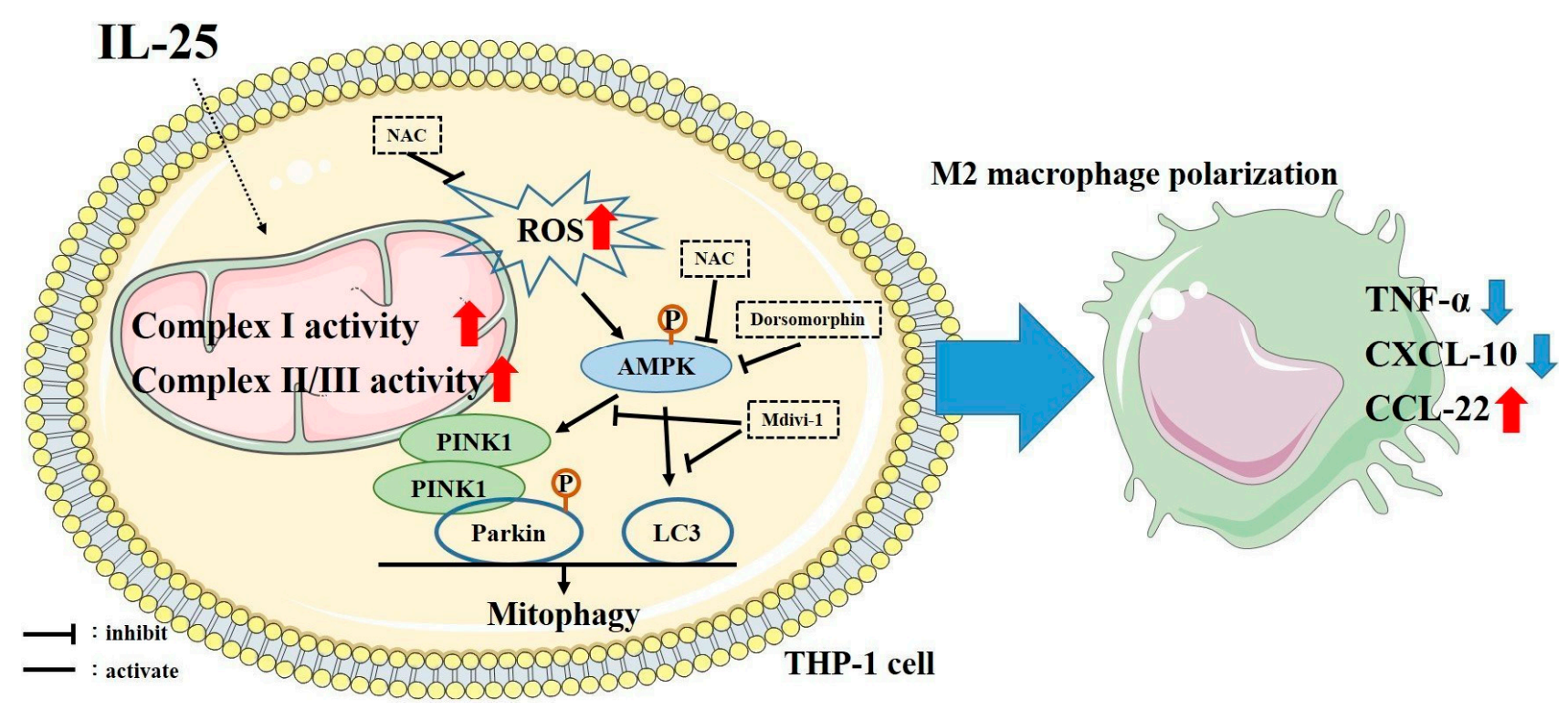

Figure 7. Schematic of the proposed intracellular mechanisms underlying IL-25-induced M2 macrophage polarization. IL-25 increased ROS production and mitochondrial complex I and complex II/III activity. Subsequently, activation of mitophagy, and then shifted M1/M2 chemokine expression via the AMPK signaling pathway in human monocyte cell line. 


\section{Materials and Methods}

\subsection{Cell Culture}

The THP-1 human monocyte cell line (American Type Culture Collection, Rockville, MD, USA) was cultured in RPMI-1640 medium containing 10\% fetal bovine serum and antibiotic-antimycotic (Gibco, Carlsbad, CA, USA, \#15240-062) in a humidified incubator at $37{ }^{\circ} \mathrm{C}$ and $5 \% \mathrm{CO}_{2}$. The cells were cultured with $20 \mathrm{ng} / \mathrm{mL}$ phorbol-12-myristate 13-acetate (PMA, Sigma-Aldrich, Saint Luis, MO, USA, \#P8139) at a concentration of $5 \times 10^{5}$ cells $/ \mathrm{mL}$ in 12 -well plates or $2 \times 10^{6}$ cells $/ \mathrm{mL}$ in 6 -well plates for $24 \mathrm{~h}$ for differentiation into macrophages. THP-1 cells were resuspended in fresh medium at a concentration of $2 \times 10^{5}$ cells $/ \mathrm{mL}$ in 24 -well plates or $1 \times 10^{6}$ cells $/ \mathrm{mL}$ in 6 -well plates overnight. Cells were stimulated with or without IL-25 $(2,10$, and $40 \mathrm{ng} / \mathrm{mL})($ PEPROTECH, East Windsor, NJ, USA, \#200-24) at different time points.

\subsection{Measurement of ROS Production}

2'-7'-Dichlorofluorescein diacetate (DCFH-DA, Sigma-Aldrich, \#D6883) was used to measure ROS production by flow cytometry.

The highly fluorescent compound $2^{\prime}, 7^{\prime}$-dichlorofluorescein (DCF) was oxidized by ROS from nonspecific esterase-cleaved DCFH-DA. THP-1 cells and THP-1 derived macrophages were incubated with $5 \mu \mathrm{M}$ DCFH-DA at $37{ }^{\circ} \mathrm{C}$ for $10 \mathrm{~min}$ before IL-25 treatment or pretreated with N-acetylcysteine (NAC, \#A7250), vitamin C (\#A5960), antimycin A (\#A8674), MitoTEMPO (\#SML0737), AMPK inhibitor dorsomorphin (\#P5499), or mdivi-1 (\#M0199) (all from purchased Sigma-Aldrich) for $30 \mathrm{~min}$. Antimycin A, a mitochondrial respiratory chain III inhibitor, interrupts the electron transfer from the cytochrome $b$ to the Qi-site, disrupting the mitochondrial Q-cycle of enzyme turnover [50]. MitoTEMPO, a triphenylphosphonium derivative, is easier to pass through all biological membranes and accumulate within mitochondria than non-targeted parent antioxidants [51].

Then, cells were washed twice with PBS. As stated above, intracellular $\mathrm{H}_{2} \mathrm{O}_{2}$ or lowmolecular-weight peroxides can oxidize DCFH-DA to the highly fluorescent compound DCF. Signals were detected with a $525 \mathrm{~nm}$ bandpass filter (FITC) using an LSR II flow cytometer (Becton Dickinson, San Jose, CA, USA).

\subsection{Measurement of Mitochondrial Complex Activity}

The mitochondrial fraction was isolated from THP-1 cells and THP-1 derived macrophages using a mitochondria isolation kit (\#ab110170), followed by freeze/thaw steps (dipping the vial into liquid nitrogen for $1 \mathrm{~min}$ and then $37^{\circ} \mathrm{C}$ until thawing) and centrifugation at a higher speed to isolate mitochondria. The activity of complex I in whole cell lysates of THP-1 cells or THP-1 derived macrophages was measured using a complex I enzyme activity microplate assay kit (\#ab109721) by following the oxidation of NADH to NAD ${ }^{+}$ and the simultaneous reduction of a dye, which leads to increased absorbance. Complex II/III activity was measured in isolated mitochondria using a MitoTox ${ }^{\mathrm{TM}}$ complex II+ III OXPHOS activity assay kit (\#ab109905). Succinate and oxidized cytochrome c were added to the mitochondria. The rate of the coupled complex II + III reactions was measured by monitoring the conversion of oxidized cytochrome $\mathrm{c}$ into reduced form, which can be observed an increase in absorbance. All assay kits including mitochondria isolation kit were purchased from Abcam (Abcam, Cambridge, MA, USA).

\subsection{Western Blotting}

After $8 \mathrm{~h}$ of IL-25 treatment or pretreatment with the NAC, rotenone (Sigma-Aldrich, \#R8875), antimycin A, AMPK, or Mdivi-1, THP-1 cells and THP-1 derived macrophages were lysed with equal volumes of ice-cold lysis buffer. Equal amounts of cell lysates were separated using SDS-PAGE and transferred to PVDF membranes. After blocking in PBS containing $5 \%$ milk and $0.1 \%$ Tween 20 , the membrane was incubated with anti-phospho (p)5'-AMP-activated protein kinase alpha (AMPK $\alpha$, \#2535), anti-AMPK $\alpha$ (\#5832), anti-PINK1 (\#6946), anti-GAPDH antibodies (\#2118S) (all from purchased Cell Signaling Technology, 
Danvers, MA), anti-p-Parkin (Abcam, Cambridge, MA, \#ab73015), anti-LC3 (GeneTex, CA, USA, \#GTX127375), or anti-Parkin (Abcam, \#ab77924) to further investigate the influence of IL-25 on mitophagy. Immunoreactive bands were visualized using a horseradish peroxidase-conjugated secondary antibody and an enhanced chemiluminescence (ECL) system (Merck Millipore, Darmstadt, Germany, \#WBKLS0500). The assay was performed by using the protocols recommended by the manufacturer. After chemiluminescent detection of phosphoproteins, we used Gentle Review ${ }^{\mathrm{TM}}$ Stripping Buffer (VWR LIFE SCIENCE, Pennsylvania, USA, \#N552) to strip the PVDF membrane. We added $10 \mathrm{~mL}$ stripping buffer to incubate the membrane with gentle sharking for $30 \mathrm{~min}$ at room temperature.

\subsection{Confocal Immunofluorescence Microscopy}

The non-adhesive THP- 1 cells were cultured with PMA in 12 wells containing a coverslip and differentiated to adhesive cells, macrophages. After IL-25 treatment, THP-1 cells and THP-1 derived macrophages were stained with MitoTracker Deep Red (Invitrogen, Waltham, MA, USA, \#M22426) $400 \mathrm{nM}$ for $30 \mathrm{~min}$ at $37^{\circ} \mathrm{C}$. After MitoTracker staining, the THP-1 cells were collected in $1.5 \mathrm{~mL}$ eppendorf, and fixed with $4 \%$ paraformaldehyde, permeabilized with $0.2 \%$ Triton X-100, blocked with PBS containing $1 \%$ BSA, and finally incubated with the anti-LC3 antibody (1:100) in blocking buffer at $4{ }^{\circ} \mathrm{C}$ overnight with end-to-end rotation. After washing, the cells were incubated with a fluorescein-conjugated goat anti-rabbit antibody (1:100, Vector Laboratories Inc., Burlingame, CA, USA, \#A11008) in a blocking buffer with end-to-end rotation. After another wash, the cells were stained with the nuclear stain 4',6-diamidino-2-phenylindole (DAPI) (300 nM; Invitrogen, \#D1306) in PBS. Finally, the cells were centrifuged at $1000 \mathrm{rpm}$ for $5 \mathrm{~min}$ by Shandon Cytospin Cytocentrifuge (Thermo Fisher Scientific, Waltham, MA, USA), mounted with ProLong ${ }^{\mathrm{TM}}$ Gold antifade mounting medium (Invitrogen, \#P36930), and covered with glass coverslips. After MitoTracker staining, THP-1 derived macrophage on a coverslip in 12 wells plate were stained following the step described above with an orbital shaker. After mounted, we imaged using an LSM 700 and a $63 \times / 1.4$ NA objective lens. (Carl Zeiss Microscopy, Göttingen, Germany).

\subsection{Enzyme-Linked Immunosorbent Assay (ELISA)}

After pretreatment with IL-25 for $2 \mathrm{~h}$, THP-1 cells were cultured with $10 \mathrm{ng} / \mathrm{mL}$ PMA, PMA combined with $10 \mathrm{ng} / \mathrm{mL}$ human IFN- $\gamma$ recombinant protein (PEPROTECH, \#300-02) and $10 \mathrm{ng} / \mathrm{mL}$ lipopolysaccharides (Sigma-Aldrich, \#L2880) or PMA combined with $10 \mathrm{ng} / \mathrm{mL}$ human IL-4 recombinant protein (PEPROTECH, \#200-04) for differentiation into macrophages, M1 macrophages or M2 macrophages. The TNF- $\alpha$ (\#DY210), CXCL-10 (\#DY266), and CCL-22 (\#DY336) concentrations of cell supernatants were collected at $48 \mathrm{~h}$ and measured using a commercial ELISA kit. (R \& D Systems, Minneapolis, MN, USA). Mdivi-1, mitochondrial division inhibitor-1, is a selective inhibitor of Drp1 that leads to mitochondrial fission and mitophagy [52,53]. The CCL-22 concentration in the supernatant of IL-25-treated macrophages and M2 macrophages treated with or without Mdivi-1 was measured. CCL-22 concentrations in THP-1 and PMA/IL-4-primed THP-1 cells treated with or without the mitophagy inhibitor Mdivi-1 were detected by ELISA.

\subsection{PINK1 Knockdown}

THP-1 cells were transduced with multiplicity of infection (MOI) 1 of shRNA lentiviral particles encoding either a non-targeting (nt) shRNA (\#TRCN0000208001) or shRNA targeting PINK1 (\#TRCN0000007097 (targeting in 3' UTR), \#TRCN0000007101, and \#TRCN0000199446 (both targeting in CDS)), and spin cells at $2250 \mathrm{rpm}$ for $30 \mathrm{~min}$ at $37^{\circ} \mathrm{C}$ according to the protocol of the RNAi core facility. In addition, THP-1 derived macrophages were also transduced with multiplicity of infection (MOI) 1 of shRNA lentiviral particles as described here above. A multiplicity of infection (MOI) is the number of transducing lentiviral particles per cell. All shRNA lentiviral particles were purchased from the RNA technology platform and gene manipulation core of Academia Sinica. According to the manual instruction of 
the RNAi core facility, the cells that transduced with more than MOI 3 would have had an off-target effect according to the analysis of RNA sequencing. In this study, we transduced shRNA lentiviral particles to THP-1 cells only with MOI 1 condition, which could almost avoid the off-target effect resulted from gene knockdown by RNA interference. For the selection of successfully transduced cells, puromycin (final concentration $0.5 \mu \mathrm{g} / \mathrm{mL}$ ) was used. After 3 d, PINK1 protein expression in PINK1 shRNA (\#TRCN0000007097 and \#TRCN0000199446)-transduced cells were both reduced by $40 \%$ compared to that in $\mathrm{nt}$ shRNA-transduced cells via Western blot. According to the outcome of knockdown efficiency by Western blotting, we used TRCN0000007097 and TRCN0000199446 for further investigation of the effects of IL-25 on ROS production, subsequent mitophagy, and CCL-22 concentration of M2 macrophages. PINK1 knockdown cells were pretreated with IL-25 for $2 \mathrm{~h}$ and differentiated into M2 macrophages. After $48 \mathrm{~h}$, the supernatant was collected, and the CCL-22 concentration was measured.

\subsection{Statistical Analysis}

All data are presented as the means \pm standard deviations. The Mann-Whitney U-test was used in each independent experiment to analyze the difference between the experimental and control groups. The densitometric data from Western blot analysis were analyzed using ImageJ software (National Institutes of Health, Bethesda, Maryland, USA) to measure the optical density of each band. The mean fluorescence intensity (MFI) of intracellular ROS level was analyzed using FCS Express 4 Flow Research (De Novo Software, Pasadena, USA) All data were analyzed using GraphPad Prism version 5.0 software (GraphPad Software Inc., San Diego, CA, USA) to determine differences between groups. A $p$ value $<0.05$ was considered to indicate a significant difference.

Author Contributions: Conceptualization: M.-L.T., W.-T.L., Y.-C.L. (Yi-Ching Lin), and C.-H.H.; methodology: M.-L.T. and C.-H.H.; formal analysis: Y.-G.T., Y.-C.L. (Yu-Chih Lin), and Y.-L.H.; investigation: W.-T.L. and Y.-C.L. (Yi-Ching Lin); data curation: Y.-T.C. and M.-K.T.; writing-original draft preparation: M.-L.T.; writing-reviewing and editing: W.-T.L., Y.-C.L. (Yi-Ching Lin), and C.H.H. All authors reviewed the manuscript. W.-T.L., Y.-C.L. (Yi-Ching Lin), and C.-H.H. contributed equally. All authors have read and agreed to the published version of the manuscript.

Funding: The study was supported by grants from the Ministry of Science and Technology of the Republic of China (MOST107-2635-B-037-003-; MOST108-2314-B-037-071-), the Kaohsiung Medical University Research Foundation (103-CCH-KMU-005), Kaohsiung Medical University Hospital Research Foundation (KMUH107-7R86), Kaohsiung Municipal Siaogang Hospital Research Foundation (S-109-05; I109-02) and the Research Center for Environmental Medicine (KMU-TC109A01-1), Kaohsiung Medical University from the Featured Areas Research Center Program within the framework of the Higher Education Sprout Project by the Ministry of Education (MOE) in Taiwan.

Institutional Review Board Statement: Not applicable.

Informed Consent Statement: Not applicable.

Data Availability Statement: The data presented in this study are available on request from the corresponding author. Data may be available upon request to interested researchers. Please send data requests to Chih-Hsing Hung, MD, PhD. Department of Pediatrics, Kaohsiung Medical University Hospital, Kaohsiung Medical University.

Acknowledgments: We thank the Center for Research Resources and Development of Kaohsiung Medical University for providing the service of LSRII and LSM 700 microscopy.

Conflicts of Interest: All authors declare no conflict of interest in relation to the work and the manuscript. The funders had no role in the design of the study; in the collection, analyses, or interpretation of data; in the writing of the manuscript, or in the decision to publish the results. 


\section{References}

1. Saenz, S.A.; Taylor, B.C.; Artis, D. Welcome to the neighborhood: Epithelial cell-derived cytokines license innate and adaptive immune responses at mucosal sites. Immunol. Rev. 2008, 226, 172-190. [CrossRef] [PubMed]

2. Fux, M.; Pecaric-Petkovic, T.; Odermatt, A.; Hausmann, O.V.; Lorentz, A.; Bischoff, S.C.; Virchow, J.C.; Dahinden, C.A. IL-33 is a mediator rather than a trigger of the acute allergic response in humans. Allergy 2014, 69, 216-222. [CrossRef] [PubMed]

3. Kabata, H.; Moro, K.; Koyasu, S.; Asano, K. Group 2 innate lymphoid cells and asthma. Allergol. Int. 2015, 64, 227-234. [CrossRef]

4. Divekar, R.; Kita, H. Recent advances in epithelium-derived cytokines (IL-33, IL-25, and thymic stromal lymphopoietin) and allergic inflammation. Curr. Opin. Allergy Clin. Immunol. 2015, 15, 98-103. [CrossRef]

5. Fort, M.M.; Cheung, J.; Yen, D.; Li, J.; Zurawski, S.M.; Lo, S.; Menon, S.; Clifford, T.; Hunte, B.; Lesley, R.; et al. IL-25 induces IL-4, IL-5, and IL-13 and Th2-associated pathologies in vivo. Immunity 2001, 15, 985-995. [CrossRef]

6. Hams, E.; Armstrong, M.E.; Barlow, J.L.; Saunders, S.P.; Schwartz, C.; Cooke, G.; Fahy, R.J.; Crotty, T.B.; Hirani, N.; Flynn, R.J.; et al. IL-25 and type 2 innate lymphoid cells induce pulmonary fibrosis. Proc. Natl. Acad. Sci. USA 2014, 111, 367-372. [CrossRef]

7. Zuo, L.; Otenbaker, N.P.; Rose, B.A.; Salisbury, K.S. Molecular mechanisms of reactive oxygen species-related pulmonary inflammation and asthma. Mol. Immunol. 2013, 56, 57-63. [CrossRef]

8. Lee, I.T.; Yang, C.M. Role of NADPH oxidase/ROS in pro-inflammatory mediators-induced airway and pulmonary diseases. Biochem. Pharmacol. 2012, 84, 581-590. [CrossRef] [PubMed]

9. Hamacher-Brady, A.; Brady, N.R. Mitophagy programs: Mechanisms and physiological implications of mitochondrial targeting by autophagy. Cell. Mol. Life Sci. 2016, 73, 775-795. [CrossRef]

10. Williams, J.A.; Ding, W.X. Targeting Pink1-Parkin-mediated mitophagy for treating liver injury. Pharmacol. Res. 2015, 102, 264-269. [CrossRef]

11. Osborn-Heaford, H.L.; Ryan, A.J.; Murthy, S.; Racila, A.M.; He, C.; Sieren, J.C.; Spitz, D.R.; Carter, A.B. Mitochondrial Rac1 GTPase import and electron transfer from cytochrome c are required for pulmonary fibrosis. J. Biol. Chem. 2012, 287, $3301-3312$. [CrossRef]

12. Carnesecchi, S.; Deffert, C.; Donati, Y.; Basset, O.; Hinz, B.; Preynat-Seauve, O.; Guichard, C.; Arbiser, J.L.; Banfi, B.; Pache, J.C.; et al. A key role for NOX4 in epithelial cell death during development of lung fibrosis. Antioxid. Redox Signal. 2011, 15, 607-619. [CrossRef]

13. Yao, X.; Sun, Y.; Wang, W.; Sun, Y. Interleukin (IL)-25: Pleiotropic roles in asthma. Respirology 2016, 21, 638-647. [CrossRef] [PubMed]

14. Held, N.M.; Houtkooper, R.H. Mitochondrial quality control pathways as determinants of metabolic health. Bioessays 2015, 37, 867-876. [CrossRef]

15. Bujak, A.L.; Crane, J.D.; Lally, J.S.; Ford, R.J.; Kang, S.J.; Rebalka, I.A.; Green, A.E.; Kemp, B.E.; Hawke, T.J.; Schertzer, J.D.; et al. AMPK activation of muscle autophagy prevents fasting-induced hypoglycemia and myopathy during aging. Cell Metab. 2015, 21, 883-890. [CrossRef] [PubMed]

16. Borowczyk, J.; Shutova, M.; Brembilla, N.C.; Boehncke, W.H. IL-25 (IL-17E) in epithelial immunology and pathophysiology. J. Allergy Clin. Immunol. 2021, 148, 40-52. [CrossRef] [PubMed]

17. Wang, Y.H.; Angkasekwinai, P.; Lu, N.; Voo, K.S.; Arima, K.; Hanabuchi, S.; Hippe, A.; Corrigan, C.J.; Dong, C.; Homey, B.; et al. IL-25 augments type 2 immune responses by enhancing the expansion and functions of TSLP-DC-activated Th2 memory cells. J. Exp. Med. 2007, 204, 1837-1847. [CrossRef]

18. Tworek, D.; Smith, S.G.; Salter, B.M.; Baatjes, A.J.; Scime, T.; Watson, R.; Obminski, C.; Gauvreau, G.M.; O’Byrne, P.M. IL-25 receptor expression on airway dendritic cells after allergen challenge in subjects with asthma. Am. J. Respir. Crit. Care Med. 2016, 193, 957-964. [CrossRef]

19. Hewitt, R.J.; Lloyd, C.M. Regulation of immune responses by the airway epithelial cell landscape. Nat. Rev. Immunol. 2021, 21, 347-362. [CrossRef] [PubMed]

20. Kirkham, P.; Rahman, I. Oxidative stress in asthma and COPD: Antioxidants as a therapeutic strategy. Pharmacol. Ther. 2006, 111, 476-494. [CrossRef]

21. Sugiura, H.; Ichinose, M. Oxidative and nitrative stress in bronchial asthma. Antioxid. Redox Signal. 2008, 10, 785-797. [CrossRef]

22. Wood, L.G.; Wark, P.A.; Garg, M.L. Antioxidant and anti-inflammatory effects of resveratrol in airway disease. Antioxid. Redox Signal. 2010, 13, 1535-1548. [CrossRef]

23. Reinmuth-Selzle, K.; Kampf, C.J.; Lucas, K.; Lang-Yona, N.; Frohlich-Nowoisky, J.; Shiraiwa, M.; Lakey, P.S.J.; Lai, S.; Liu, F.; Kunert, A.T.; et al. Air pollution and climate change effects on allergies in the anthropocene: Abundance, interaction, and modification of allergens and adjuvants. Environ. Sci. Technol. 2017, 51, 4119-4141. [CrossRef]

24. Jiang, L.; Diaz, P.T.; Best, T.M.; Stimpfl, J.N.; He, F.; Zuo, L. Molecular characterization of redox mechanisms in allergic asthma. Ann. Allergy Asthma Immunol. 2014, 113, 137-142. [CrossRef]

25. Tan, H.Y.; Wang, N.; Li, S.; Hong, M.; Wang, X.; Feng, Y. The reactive oxygen species in macrophage polarization: Reflecting its dual role in progression and treatment of human diseases. Oxid. Med. Cell. Longev. 2016, 2016, 2795090. [CrossRef] [PubMed] 
26. Beale, J.; Jayaraman, A.; Jackson, D.J.; Macintyre, J.D.R.; Edwards, M.R.; Walton, R.P.; Zhu, J.; Man Ching, Y.; Shamji, B.; Edwards, M.; et al. Rhinovirus-induced IL-25 in asthma exacerbation drives type 2 immunity and allergic pulmonary inflammation. Sci. Transl. Med. 2014, 6, 256ra134. [CrossRef] [PubMed]

27. Hong, J.Y.; Bentley, J.K.; Chung, Y.; Lei, J.; Steenrod, J.M.; Chen, Q.; Sajjan, U.S.; Hershenson, M.B. Neonatal rhinovirus induces mucous metaplasia and airways hyperresponsiveness through IL-25 and type 2 innate lymphoid cells. J. Allergy Clin. Immunol. 2014, 134, 429-439. [CrossRef]

28. Petersen, B.C.; Dolgachev, V.; Rasky, A.; Lukacs, N.W. IL-17E (IL-25) and IL-17RB promote respiratory syncytial virus-induced pulmonary disease. J. Leukoc. Biol. 2014, 95, 809-815. [CrossRef]

29. Aguilera-Aguirre, L.; Bacsi, A.; Saavedra-Molina, A.; Kurosky, A.; Sur, S.; Boldogh, I. Mitochondrial dysfunction increases allergic airway inflammation. J. Immunol. 2009, 183, 5379-5387. [CrossRef] [PubMed]

30. St-Pierre, J.; Buckingham, J.A.; Roebuck, S.J.; Brand, M.D. Topology of superoxide production from different sites in the mitochondrial electron transport chain. J. Biol. Chem. 2002, 277, 44784-44790. [CrossRef]

31. Quinlan, C.L.; Orr, A.L.; Perevoshchikova, I.V.; Treberg, J.R.; Ackrell, B.A.; Brand, M.D. Mitochondrial complex II can generate reactive oxygen species at high rates in both the forward and reverse reactions. J. Biol. Chem. 2012, 287, 27255-27264. [CrossRef]

32. Paradies, G.; Petrosillo, G.; Pistolese, M.; Di Venosa, N.; Federici, A.; Ruggiero, F.M. Decrease in mitochondrial complex I activity in ischemic/reperfused rat heart: Involvement of reactive oxygen species and cardiolipin. Circ. Res. 2004, 94, 53-59. [CrossRef]

33. Redout, E.M.; Wagner, M.J.; Zuidwijk, M.J.; Boer, C.; Musters, R.J.; van Hardeveld, C.; Paulus, W.J.; Simonides, W.S. Rightventricular failure is associated with increased mitochondrial complex II activity and production of reactive oxygen species. Cardiovasc. Res. 2007, 75, 770-781. [CrossRef] [PubMed]

34. Sanchez-Duarte, E.; Cortes-Rojo, C.; Sanchez-Briones, L.A.; Campos-Garcia, J.; Saavedra-Molina, A.; Delgado-Enciso, I.; LopezLemus, U.A.; Montoya-Perez, R. Nicorandil affects mitochondrial respiratory chain function by increasing complex III activity and ROS production in skeletal muscle mitochondria. J. Membr. Biol. 2020, 253, 309-318. [CrossRef] [PubMed]

35. Parsons, M.J.; Green, D.R. Mitochondria in cell death. Essays Biochem. 2010, 47, 99-114. [PubMed]

36. Valente, E.M.; Abou-Sleiman, P.M.; Caputo, V.; Muqit, M.M.; Harvey, K.; Gispert, S.; Ali, Z.; Del Turco, D.; Bentivoglio, A.R.; Healy, D.G.; et al. Hereditary early-onset Parkinson's disease caused by mutations in PINK1. Science 2004, 304, 1158-1160. [CrossRef]

37. Kitada, T.; Asakawa, S.; Hattori, N.; Matsumine, H.; Yamamura, Y.; Minoshima, S.; Yokochi, M.; Mizuno, Y.; Shimizu, N. Mutations in the parkin gene cause autosomal recessive juvenile parkinsonism. Nature 1998, 392, 605-608. [CrossRef]

38. Bingol, B.; Sheng, M. Mechanisms of mitophagy: PINK1, Parkin, USP30 and beyond. Free Radic. Biol. Med. 2016, 100, $210-222$. [CrossRef] [PubMed]

39. Zhuang, N.; Li, L.; Chen, S.; Wang, T. PINK1-dependent phosphorylation of PINK1 and Parkin is essential for mitochondrial quality control. Cell Death Dis. 2016, 7, e2501. [CrossRef]

40. Patel, A.S.; Song, J.W.; Chu, S.G.; Mizumura, K.; Osorio, J.C.; Shi, Y.; El-Chemaly, S.; Lee, C.G.; Rosas, I.O.; Elias, J.A.; et al. Epithelial cell mitochondrial dysfunction and PINK1 are induced by transforming growth factor-beta1 in pulmonary fibrosis. PLoS ONE 2015, 10, e0121246. [CrossRef]

41. Garcia, D.; Shaw, R.J. AMPK: Mechanisms of cellular energy sensing and restoration of metabolic balance. Mol. Cell 2017, 66, 789-800. [CrossRef] [PubMed]

42. Wu, S.; Zou, M.H. AMPK, Mitochondrial function, and cardiovascular disease. Int. J. Mol. Sci. 2020, 21, 4987. [CrossRef] [PubMed]

43. Zou, M.H.; Kirkpatrick, S.S.; Davis, B.J.; Nelson, J.S.; Wiles, W.G.T.; Schlattner, U.; Neumann, D.; Brownlee, M.; Freeman, M.B.; Goldman, M.H. Activation of the AMP-activated protein kinase by the anti-diabetic drug metformin in vivo. Role of mitochondrial reactive nitrogen species. J. Biol. Chem. 2004, 279, 43940-43951. [CrossRef] [PubMed]

44. Rabinovitch, R.C.; Samborska, B.; Faubert, B.; Ma, E.H.; Gravel, S.P.; Andrzejewski, S.; Raissi, T.C.; Pause, A.; St-Pierre, J.; Jones, R.G. AMPK maintains cellular metabolic homeostasis through regulation of mitochondrial reactive oxygen species. Cell Rep. 2017, 21, 1-9. [CrossRef] [PubMed]

45. Han, Y.; Wang, Q.; Song, P.; Zhu, Y.; Zou, M.H. Redox regulation of the AMP-activated protein kinase. PLoS ONE 2010, 5, e15420. [CrossRef]

46. Herzig, S.; Shaw, R.J. AMPK: Guardian of metabolism and mitochondrial homeostasis. Nat. Rev. Mol. Cell Biol. 2018, 19, 121-135. [CrossRef]

47. Irrcher, I.; Adhihetty, P.J.; Sheehan, T.; Joseph, A.M.; Hood, D.A. PPARgamma coactivator-1alpha expression during thyroid hormone- and contractile activity-induced mitochondrial adaptations. Am. J. Physiol. Cell Physiol. 2003, 284, C1669-C1677. [CrossRef]

48. Jornayvaz, F.R.; Shulman, G.I. Regulation of mitochondrial biogenesis. Essays Biochem. 2010, 47, 69-84.

49. Feng, J.; Li, L.; Ou, Z.; Li, Q.; Gong, B.; Zhao, Z.; Qi, W.; Zhou, T.; Zhong, J.; Cai, W.; et al. IL-25 stimulates M2 macrophage polarization and thereby promotes mitochondrial respiratory capacity and lipolysis in adipose tissues against obesity. Cell. Immunol. 2018, 15, 493-505. [CrossRef]

50. Ward, J.P. Point: Hypoxic pulmonary vasoconstriction is mediated by increased production of reactive oxygen species. J. Appl. Physiol. 2006, 101, 993-995, discussion 999. [CrossRef] 
51. Jin, H.; Kanthasamy, A.; Ghosh, A.; Anantharam, V.; Kalyanaraman, B.; Kanthasamy, A.G. Mitochondria-targeted antioxidants for treatment of Parkinson's disease: Preclinical and clinical outcomes. Biochim. Biophys. Acta 2014, 1842, 1282-1294. [CrossRef] [PubMed]

52. Luo, X.; Liu, R.; Zhang, Z.; Chen, Z.; He, J.; Liu, Y. Mitochondrial division inhibitor 1 attenuates mitophagy in a rat model of acute lung injury. Biomed. Res. Int. 2019, 2019, 2193706. [CrossRef] [PubMed]

53. Lin, X.H.; Qiu, B.Q.; Ma, M.; Zhang, R.; Hsu, S.J.; Liu, H.H.; Chen, J.; Gao, D.M.; Cui, J.F.; Ren, Z.G.; et al. Suppressing DRP1-mediated mitochondrial fission and mitophagy increases mitochondrial apoptosis of hepatocellular carcinoma cells in the setting of hypoxia. Oncogenesis 2020, 9, 67. [CrossRef] [PubMed] 